\title{
Keratoprostheses for corneal blindness: a review of contemporary devices
}

\author{
This article was published in the following Dove Press journal: \\ Clinical Ophthalmology \\ 16 April 2015 \\ Number of times this article has been viewed
}

\author{
Venkata S Avadhanam ${ }^{1,2}$ \\ Helen E Smith ${ }^{2}$ \\ Christopher Liu ${ }^{1-3}$ \\ 'Sussex Eye Hospital, ${ }^{2}$ Brighton \\ and Sussex Medical School, Brighton, \\ ${ }^{3}$ Tongdean Eye Clinic, Hove, UK
}

\begin{abstract}
According to the World Health Organization, globally 4.9 million are blind due to corneal pathology. Corneal transplantation is successful and curative of the blindness for a majority of these cases. However, it is less successful in a number of diseases that produce corneal neovascularization, dry ocular surface and recurrent inflammation, or infections. A keratoprosthesis or KPro is the only alternative to restore vision when corneal graft is a doomed failure. Although a number of KPros have been proposed, only two devices, Boston type-1 KPro and osteo-odonto-KPro, have came to the fore. The former is totally synthetic and the latter is semi-biological in constitution. These two KPros have different surgical techniques and indications. Keratoprosthetic surgery is complex and should only be undertaken in specialized centers, where expertise, multidisciplinary teams, and resources are available. In this article, we briefly discuss some of the prominent historical KPros and contemporary devices.
\end{abstract}

Keywords: keratoprosthesis, OOKP, KPro, Boston KPro, cornea, ocular surface

\section{Introduction}

Keratoprosthesis (KPro) is an ancient idea; it predates the origin of corneal transplantation. In the eighteenth century, guided by an expanding knowledge in human anatomy along with a surge in empirical approach to medicine, the earlier physicians were able to gradually recognize corneal diseases as a specific cause for blindness. While the clinical assessment and investigation methodologies for the disease evaluation were still nascent, interestingly, preliminary attempts for treating corneal blindness began with artificial materials as a means of corneal substitution. Sadly, these trials were plagued with severe failures mainly due to the lack of parallel developments in the biomaterials, surgical instruments, and techniques. With the evolution of corneal transplantation that was more promising than the artificial implants, unsurprisingly, interest in KPros had wilted. Although corneal grafts may have a life span of some decades in low-risk cases, the visual gain may be limited by astigmatism and complications; and, even in successful cases, visual recovery following grafting can be protracted. ${ }^{1,2}$ Over the years, limitations of the corneal grafts have surfaced; it is now well recognized that they do not offer a lasting solution to corneal blindness.

A corneal transplant carries a lifetime risk of rejection and failure. Despite the improvements in corneal lamellar surgical techniques and limbal stem cell transplantation, and a widespread usage of immunosuppression, graft survival has not improved in autoimmune diseases, chemical injuries, and dry eyes. ${ }^{3-5}$ Thus, the place for KPros is well preserved. In this article, we endeavor to succinctly describe some of the prominent and contemporaneous KPros and their performance. 


\section{Need for KPros}

In a recent estimate, the World Health Organization identifies that globally 4.9 million people are bilaterally corneal blind, which accounts for $12 \%$ of the total burden of global blindness (39 million). ${ }^{6-8}$ By extrapolation of this data, it can be inferred that there could be a significant proportion of unilaterally corneal blind people living in the world at present. ${ }^{6}$ These estimates also indicate that worldwide, a large number of people could benefit from corneal transplantation.

Corneal grafting is proven to be one of the highly successful solid organ transplantations. A lamellar or full-thickness corneal graft is performed to restore vision when the host corneal transparency is lost in a disease process. During the first few years after the transplantation, corneal graft survival is generally high. The Australian Corneal Graft Registry (ACGR) and NHS Blood and Transplant authority reported a survival rate in the first year as $87 \%$ and $93 \%$, respectively. ${ }^{9,10}$ Five-year transplant survival rate for the penetrating grafts in the former was $73 \%$ and in the latter $72 \% .{ }^{9}, 10$ According to the Australian report, the survival of lamellar transplants in the first year was $80 \% .{ }^{9}$ It also reported that after 15 years, the transplant survival rates had steadily fallen to $46 \%$ for the full-thickness grafts and $41 \%$ for lamellar grafts. ${ }^{9}$

Corneal transplantation is a well-known successful procedure for a number of conditions such as keratoconus, corneal opacities, and bullous keratopathy. ${ }^{11}$ However, viability of corneal grafts in recurrent and chronic inflammatory conditions such as sicca disease states and herpetic infections, and in vascularized corneal beds is low. ${ }^{5,11}$ Keratolimbal and limbal stem cell allografts performed for conditions associated with severe limbal stem cell deficiency are also constrained by limited success and frequently require immunosuppression for survival. ${ }^{12,13}$ In a majority of these situations, a KPro is the only available option to traverse the ocular surface in order to restore vision.

Globally, availability of corneal donor material is limited, especially in developing countries. In addition, facilities for processing, preservation, storage, and supply of the transplantable tissues are a challenge for many nations, again principally for the developing countries. ${ }^{6}$ Besides, biological tissue transplants carry a risk of transmissible diseases such as blood-borne infections, Creutzfeldt-Jakob disease (CJD), tuberculosis, hepatitis $\mathrm{C}$, and venereal infections. It is pleasing to consider that an artificial cornea could eliminate the problems associated with corneal allografts; however, at present, KPro is not an alternative to routine corneal transplantation. A KPro is indicated only in end-stage corneal blindness due to conditions such as severe ocular surface disease and multiple graft failures - principally when conventional transplantation is a destined failure.

\section{History and development of KPros}

Pellier de Quengsy was the first to record his ideas of a KPro that consisted of a convex glass plate surrounded by a silver rim. ${ }^{14}$ Nussbaum described a collar-stud glass device with two plates that sandwich the corneal tissue, which are also connected in the center by an optical cylinder. ${ }^{14,15}$ He performed animal experiments with his KPro and also implanted in humans. Heuser implanted a KPro made of quartz material and implanted in humans. ${ }^{16}$ Abbate applied a skirt made of milk protein and rubber to the glass and inserted in animals. ${ }^{16}$ Although it was extruded shortly afterward, his efforts emphasized the importance of a skirt material for better tissue incorporation of the KPro. Salzer experimented with a quartz crystal enclosed in a platinum ring with prongs, and he also recognized the importance of a skirt material. ${ }^{16}$ Celluloid was used in a hat-shaped KPro model by Dimmer as a first polymeric biofunctional implant. ${ }^{14}$

Interest in synthetic corneal replacements was declined in the early part of the last century, largely due to the rise of cadaveric corneal transplantation. In addition, the existing KPros were not successful. With the breakthrough discovery of polymethylmethacrylate (PMMA), as a potentially implantable material in the eye, interest in KPro was rekindled. ${ }^{17}$ PMMA has fulfilled the need for a stable and biocompatible optic. Gradually, a two-part "core-skirt" skeleton for the KPro was recognized. ${ }^{18-20}$ Choyce and Stone developed a two-piece KPro independently. ${ }^{18,20}$

Dohlman et al introduced a collar-button model made of PMMA. ${ }^{21}$ Ruedemann devised a satellite-shaped optical piece made of silicone attached to a Dacron skirt. ${ }^{22}$ Cardona developed a two-piece nut-and-bolt KPro. ${ }^{19} \mathrm{He}$ also used a pigmented PMMA cylinder with a fenestrated Teflon skirt in a later design. ${ }^{23}$ Another essential contribution by Cardona was to project the optic through the lid in extremely dry ocular surfaces. ${ }^{23}$ Girard developed a KPro similar to Cardona's nut-and-bolt device but used Proplast as a skirt material. ${ }^{24}$ Polack developed a KPro made of a ceramic skirt, which was implanted external to the cornea. ${ }^{25}$ In some cases, the optic was also projected outside the lids. ${ }^{25}$ Caldwell developed a softer skirt material made from polyurethane. ${ }^{26}$ Worst et al prepared a rivet-shaped champagne cork KPro and sutured it to the sclera using steel wires. ${ }^{26,27}$ Pintucci et al developed a KPro made of a PMMA optic and a Dacron skirt. ${ }^{28,29}$ This device is implanted in two stages. Labial or cheek mucous membrane covers the ocular surface in stage-1. The device is buried in the submuscular pouch for 2-3 months before its implantation into the eye (Figure 1). Readers are advised to refer to Table 1 for further details on historical KPros. 


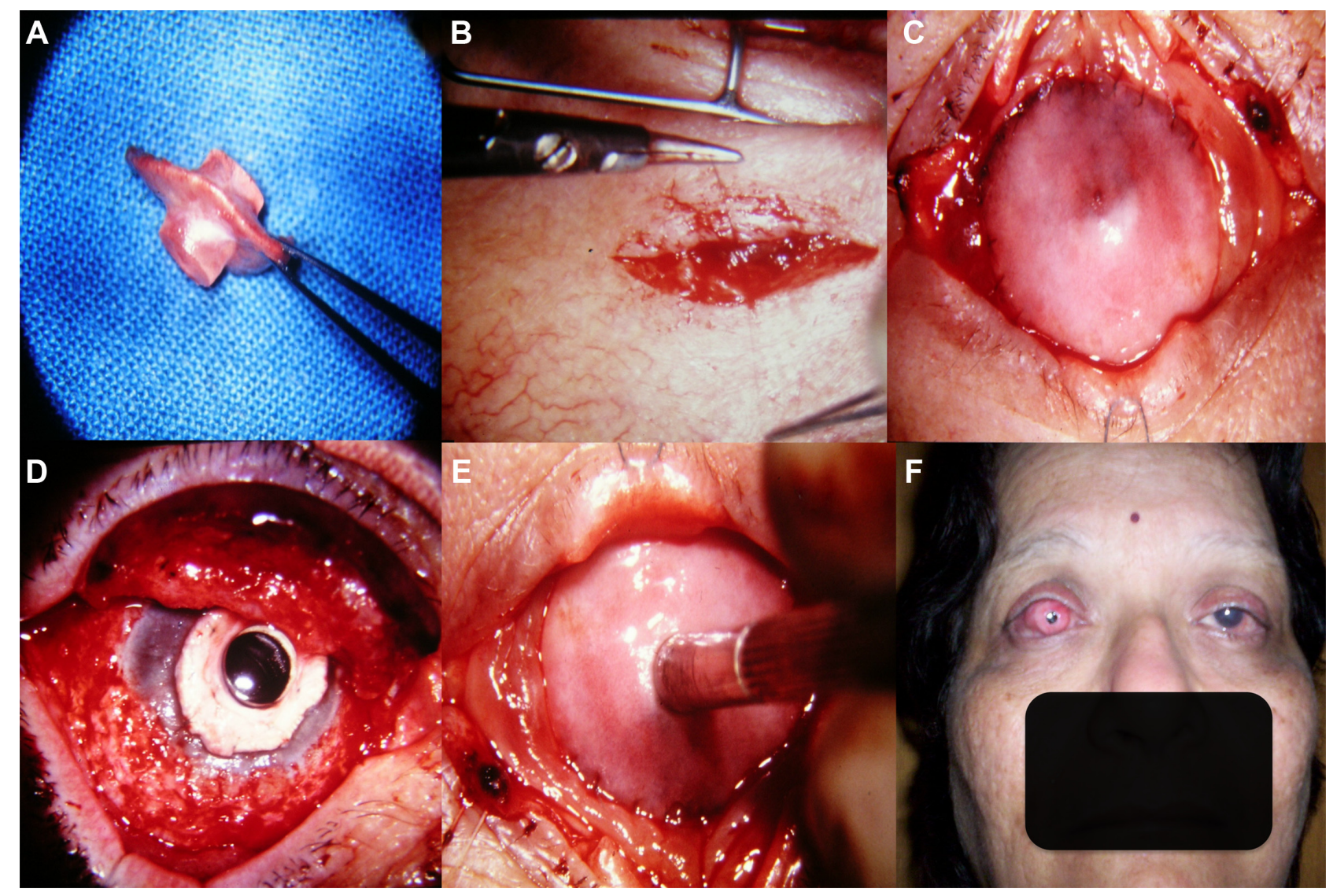

Figure I Pintucci KPro.

Notes: (A) Pintucci KPro device. (B) First stage - insertion of Pintucci device into submuscular pouch for vascular ingrowth. (C) First stage - covering the ocular surface with buccal/labial mucosa. (D) Second stage - implantation of the retrieved device into the eye (2-3 months after the stage I). (E) Second stage - creating an opening in the buccal mucosa to expose the optic. (F) Postoperative appearance of a successful Pintucci KPro. All pictures of this figure are the courtesy of Dr Qureshi Maskati. Abbreviation: KPro, keratoprosthesis.

Chirila et al noted that investigators have tried polymers other than PMMA as optical components for their KPros such as the following: silicone by Ruedemann, polysiloxane by Liebel, silicone rubber by Dohlman, polyvinyl alcohol hydrogels by Ikada, and polycarbonate by Worst, Singh, and Andel group (champagne cork KPro). ${ }^{16}$ Various porous skirts made of Proplast (Barber), Teflon (Legeais et al), hydrogels (Crawford et al), poly-2-hydroxyethyl methacrylate (Mester), and silicone-carbon (Kain) were also tried with the intention of promoting better tissue integration. ${ }^{30-34}$ All of the above devices have not shown promising results over the long term but failed with common problems such as extrusions, tissue melts, infections, and retinal detachments, which primarily arise due to the lack of permanent bio-integration. ${ }^{30,35}$

Though a variety of KPros were proposed, essentially, they can be grouped into three basic categories based on their fixation method in the eye: 1) a collar-stud device sandwiches cornea between the two skirt plates (eg, Boston KPro), 2) an intracorneal device secures the skirt inside the corneal stromal layers (eg, AlphaCor), and 3) an epicorneal device is held on the surface of the cornea and sclera (eg, osteoodonto-KPro [OOKP], Pintucci KPro). In general, these devices have a central core that is a transparent optical portion that transmits the light to the retina. Although a number of materials, models, and techniques were described, only two prostheses, the Boston type-1 KPro (Boston KPro) and OOKP, have proven successful. ${ }^{36}$ Yet, these devices necessitate multiple surgical revisions and can give rise to severe complications. Thus, the search for an ideal KPro continues, which still remains elusive. ${ }^{37}$

\section{General considerations for KPro surgery}

A KPro is indicated to restore vision in cases of corneal blindness only when corneal transplantation has failed or runs a high risk for failure. Patients being considered for a KPro should be carefully evaluated for their suitability. Often, these patients would have had received multiple corneal grafts, ocular surface, and lid reconstruction procedures 


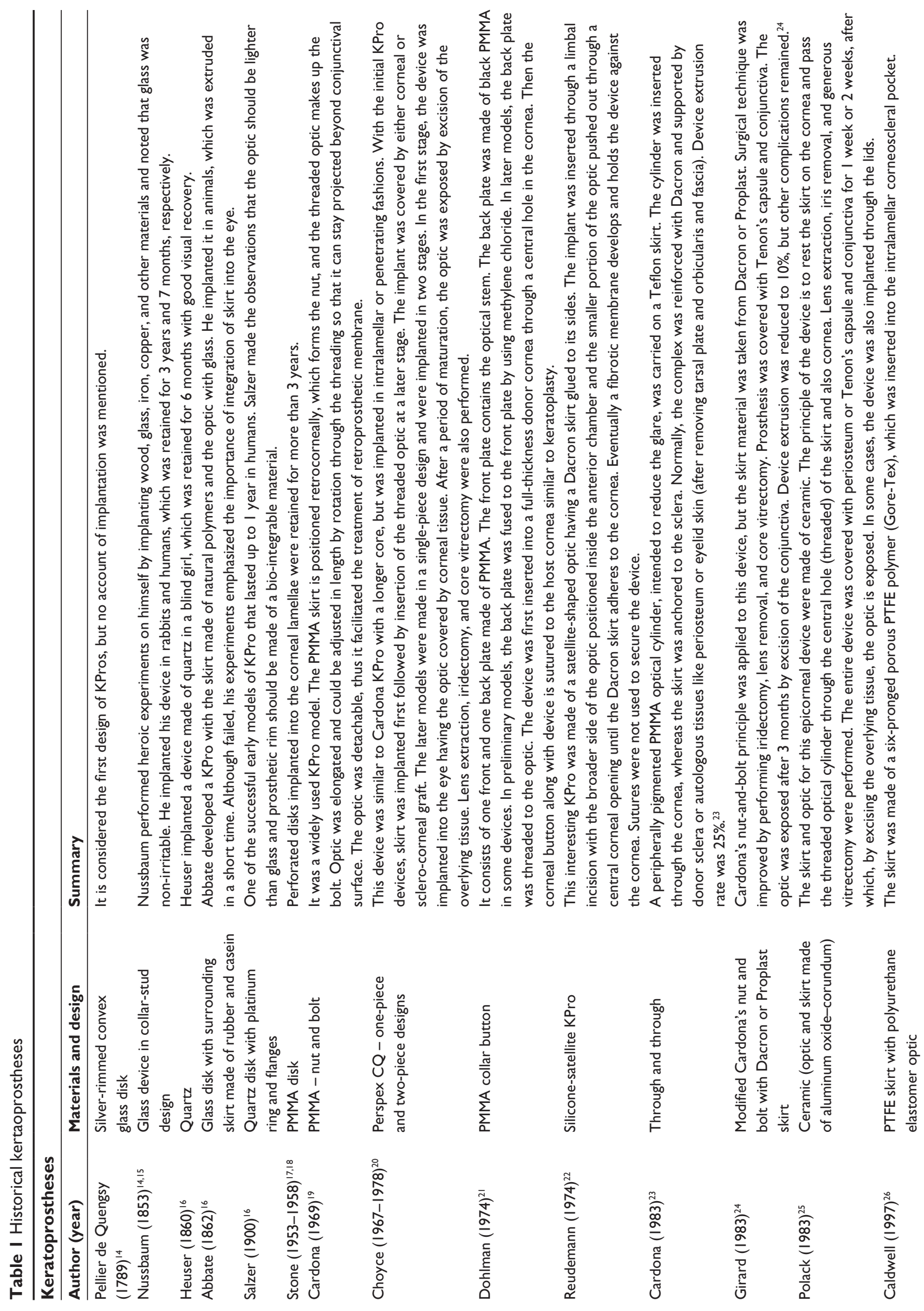



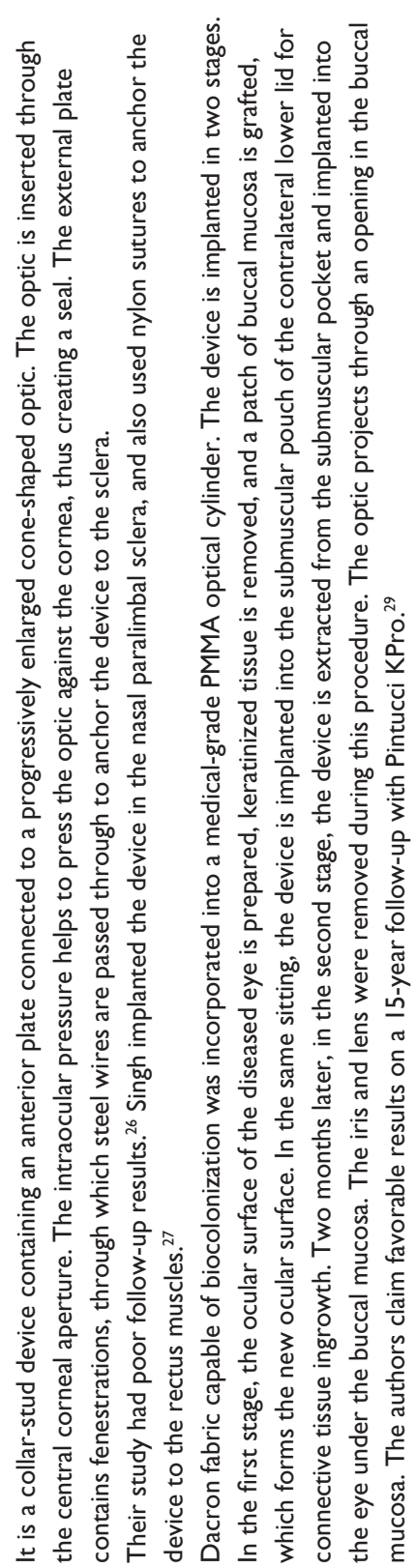

before being referred to the KPro surgeon. Hence, knowledge of the underlying diagnosis, present condition of the eye, previous treatments including the number and types of ocular surgeries and use of steroids, or immunosuppression is required for planning a management strategy. During the initial assessment, amount of surface scarring, forniceal shortening, and surface keratinization should be noted. The baseline tear secretion should also be quantified in order to determine the choice of KPro. These patients may have associated lid abnormalities, which could influence the choice of KPro and surgical approach. As a general rule, wet and blinking eyes with adequate tear production may be suitable for Boston type-1 KPro, whereas dry and keratinized eyes with impaired blinking and associated lid abnormalities should be considered for OOKP. The ability to apply and retain soft contact lens must be determined if a Boston type-1 KPro is considered..$^{15}$

Glaucoma is a frequent association with the ocular surface disease. Multiple mechanisms may contribute to the development of glaucoma: the primary disease process may involve the trabeculum, the irido-corneal angle can be narrowed by the development of synechiae, and conjunctival scarring and surface keratinization can limit episcleral venous drainage. In addition, frequent use of steroids can contribute further to the occurrence of new-onset glaucoma and progression of existing glaucoma. Detection of glaucoma (and its progression) can be difficult in ocular surface disease due to poor view of the fundus and inaccuracy in measuring intraocular pressure (IOP).

Fundal examination is usually not feasible in these patients due to opacities of the cornea and media.

An anterior segment optical coherence tomography (OCT) or ultrasound biomicroscopy should be performed to determine the status of anterior segment structures and irido-corneal angles before planning for surgery. The surgeon should look for and document the presence of corneal thinning, scars, sequelae of previous perforations, signs of previous glaucoma surgery such as glaucoma tubes and blebs, the amount of corneal neovascularization, status of the lens and iris, and evidence of vitreoretinal procedures in order to plan the necessary surgical steps to be undertaken. The presence or absence of the lens, and axial length of the globe are essential to know in order to determine the choice of Boston type-1 KPro (phakic or aphakic model), and power of the optical cylinder in OOKP surgery. ${ }^{15,38}$

Visual potential needs to be carefully estimated prior to offering surgery; especially, the presence of retinal pathology and advanced glaucoma should be excluded. B-scan 
ultrasonography must be performed to exclude retinal pathology. By and large, KPro patients cannot perform satisfactory visual field tests. The absence of light perception indicates poor prognosis, but an inaccurate light projection may be acceptable since it could be due to associated media opacities rather than posterior segment pathology. Electrophysiological tests like electroretinogram and visual-evoked potential could be beneficial in doubtful situations, but they may also not be precise in quantifying the visual potential in the presence of media opacities and bilateral blindness. ${ }^{38,39}$ History of regaining vision with previous surgical procedures is a good indication of visual recovery. In general, a KPro is offered to patients with bilaterally poor vision to the degree of hand moments or light perception. Only one eye should be offered a KPro, and the second eye should be kept as a spare and must be treated fully for glaucoma and other pathology as appropriate in order to preserve the visual potential. Patients having both eyes suitable for KPro must be carefully counseled to choose the eye for KPro implantation. When OOKP is considered, particular attention should be paid to the orodental assessment. ${ }^{38,40}$

Patient counseling for KPro surgery should be aimed at determining the visual needs and general health status of the patient and to establish patient suitability and willingness to undertake the surgery. By this stage, the surgeon would ideally have determined and discussed with the patient about the choice of KPro device and surgical procedure, and its implications. The patient counseling session in our setup is undertaken with the help of a clinical psychologist. The psychologist assesses patient's adaptation to the blindness, current life style and coping mechanisms, current employment, and social support in place. In our experience, a small number of patients have psychological morbidity, and addiction to alcohol and smoking. These patients may require psychiatric and de-addiction treatments for better outcomes. ${ }^{41}$ During the counseling, it is important to determine patient suitability for the surgery and provide verbal and written information - explaining the steps for KPro surgery and changes to the life after the surgery - and most importantly, to establish realistic expectations with the patients. Also, the counselor offers help when the patient is not psychologically ready to accept surgery but is willing for it. ${ }^{41-43}$

In some patients, the primary medical illnesses contribute to the ocular surface disease such as Stevens-Johnson syndrome (SJS), bullous pemphigoid, and graft versus host disease (GVHD). This group of patients may have associated skin lesions, and mucosal disease in the urethra, pharynx, and esophagus predisposing them to the risks of anesthesia. ${ }^{44}$ A multidisciplinary team consisting of anesthetists, physicians, orodental, oculoplastics, vitreoretinal, and glaucoma surgeons is essential for optimum outcomes. A conscientious approach to patient expectations while offering psychological and social support, and ensuring provisions for patient access to the hospital facilities in emergency and planned follow-up care is crucial for the success of the service. Patient support groups, leaflets and written information, clear instructions, and education of the patients and carers should form part of the care pathway. Implantation of the device is the first step to the patient's journey through the KPro. Usually, these patients would have subsequent procedures performed to preserve the implant on the eye and to address the arising complications with time. Because patient follow-ups and postoperative care are a lifelong process, these procedures should only be offered at the specialist institutions having sufficient work force, experience, and resources in place. ${ }^{15,40}$

\section{Soft KPros}

In the recent past, a few devices based on soft polymers have progressed from the laboratory studies to human trials. The skirts and optics of these devices are made of softer materials.

\section{AlphaCor KPro}

The Lions Eye Institute in Australia developed a bio-integrable keratoprosthetic device made of poly-2-hydroxyethyl methacrylate, otherwise known as the Chirila KPro. ${ }^{45}$ It was first implanted in humans in 1998 and was subsequently approved by the US Food and Drug Administration (FDA) in 2003. ${ }^{46}$ Grossly, it resembles the natural cornea by having a central clear zone and a peripheral skirt. The central clear optical part is manufactured with reduced water content in the hydrogel, while the peripheral skirt is made of the same polymer but with a high water content. An interpenetrating network of polymers binds the two differing polymeric zones of the same compound. ${ }^{47}$ The main principle behind this device is that the outer skirt is integrable with the corneal tissue by the invasion of keratocytes, while the central zone remains optically clear (Figure 2A).

The AlphaCor is indicated when a corneal graft is perceived as a high risk for failure as in cases with a history of prior graft failures. The device is generally offered only when the vision is reduced to $6 / 60$ or light perception. There should be good retinal and optic nerve functions for visual regain. Advanced glaucoma is a relative contraindication. ${ }^{15}$ The AlphaCor is generally suitable for eyes with adequate 

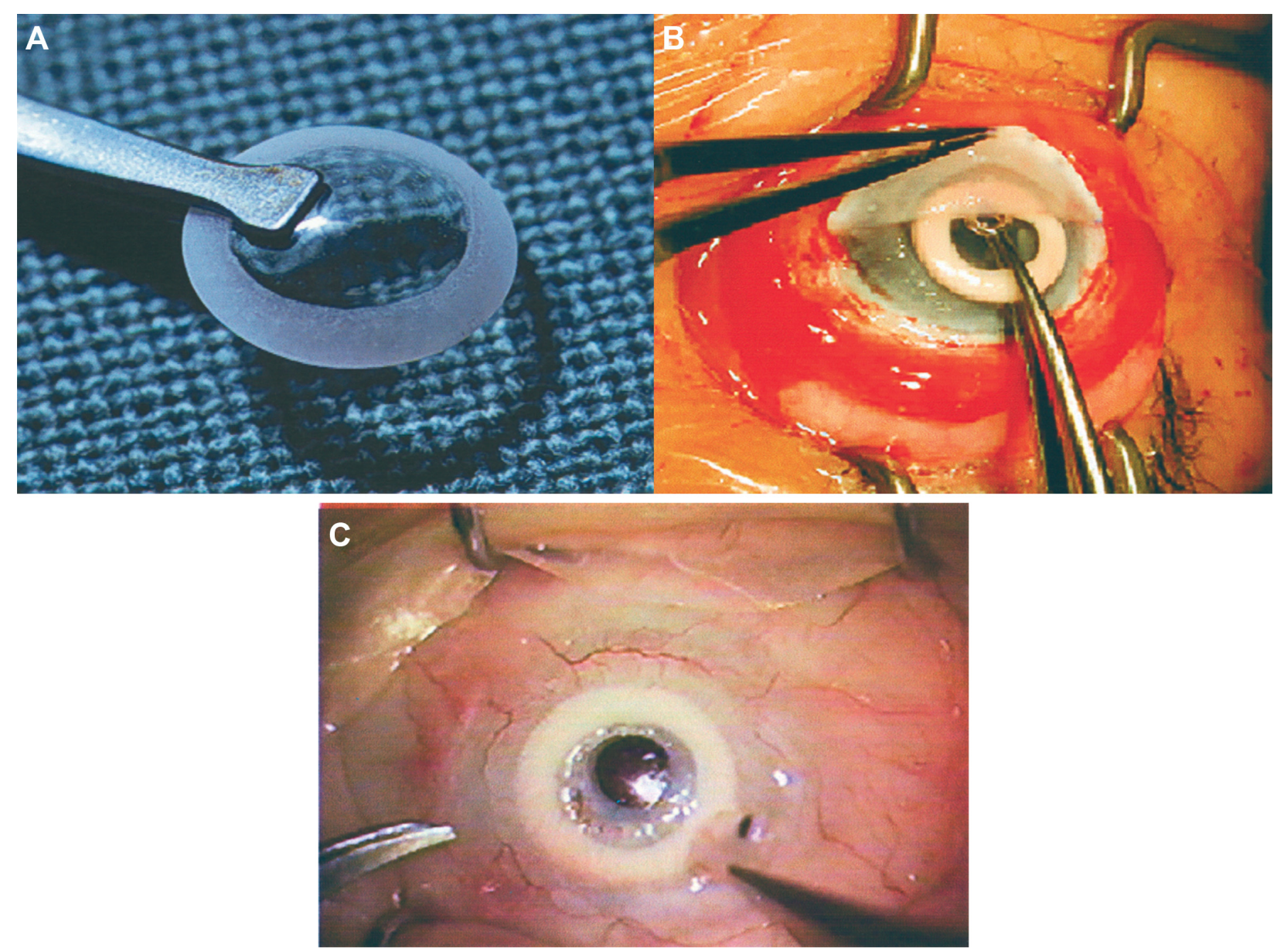

Figure 2 AlphaCor keratoprosthesis.

Notes: (A) AlphaCor device. (B) First stage - insertion of AlphaCor within the corneal lamellar pocket. A 3 mm central zone part of the posterior lamella is trephined. (C) Second stage - the external portion of the optic is exposed by excision of the superior corneal lamella. Adapted by permission from Macmillan Publishers Ltd: Eye. Hicks CR, Crawford G], Lou X, et al. Corneal replacement using a synthetic hydrogel cornea, AlphaCor[trade]: device, preliminary outcomes and complications. 2003; I7(3):385-392, Copyright @2003. ${ }^{109}$

tear secretion. This device is supplied for aphakic, pseudophakic, and phakic eyes separately. The survival of the device is poor in the presence of an active inflammation and recurrent herpes infection; hence; it should be avoided in those situations. ${ }^{48}$

AlphaCor device is implanted intrastromally into the cornea in two stages. In the first stage, a lamellar dissection is carried out from the superior limbus to create a lamellar pocket. An entry wound of $180^{\circ} \mathrm{C}$ width is created for access. The anterior and posterior lamellae are split into approximately $50 \%$ thickness layers of each. The posterior layer is trephined with a $3.5 \mathrm{~mm}$ diameter trephine in the center to reach the anterior chamber. Then the prosthesis is inserted in between the two lamellae, and the superior layer is closed with interrupted sutures. At this stage, a conjunctival tissue flap can be overlaid if the corneal surface is uneven. Two to three months later, the second-stage procedure is performed with the anticipation that the host stromal fibroblasts would have grown into the matrix of the prosthesis. A $3.5 \mathrm{~mm}$ dermatological trephine is used to create an aperture through the anterior lamella, which completes the procedure and enables the patient to see through the prosthesis (Figure $2 \mathrm{~B}$ and $\mathrm{C}$ ). ${ }^{46}$

The AlphaCor has retention rates of $80 \%$ and $62 \%$ after the first and second years of follow-up, respectively, as found in a retrospective study of 322 cases by Hicks et al. ${ }^{46}$ They recommend an indefinite use of topical medroxyprogesterone to enhance device retention. Despite its usage, stromal melts have occurred in $27 \%$ of their cases, out of which, $65 \%$ have resulted in device explantation. ${ }^{46}$ In a review involving 15 AlphaCor implants, Jiraskova et al have reported a survival rate of $87 \%$ in the first year, $58 \%$ in the second, and $42 \%$ in the third year of follow-up. ${ }^{48}$ Stromal melts occurred in $60 \%$ cases leading to device explanation in $33 \%$ of the total cases. ${ }^{48}$ Although the device is retained well in the first few years, the visual regain was affected by the occurrence of deposits on the optic and surface spoliation of the device 
in up to $8.4 \%-20 \%$ of cases. ${ }^{46,48}$ Ngakeng et al have chosen to avoid the second stage of the procedure and left the surface layer of the cornea intact, since the majority of the complications happen after the stage $2 .{ }^{49}$ They reported a short series of six cases without a stage 2 , and found no corneal melts or device extrusions at a follow-up range of 14-38 months. Conceivably, the visual regain was compromised with their approach, but they reported an improvement in postoperative comfort and a reduction of complications. Owing to the risks of complications coupled with poor visual results and a growing popularity of the Boston KPro, the AlphaCor implant is a not a favored option nowadays. ${ }^{15}$

\section{Legeais BioKPro-III}

Legeais BioKPro-III is a soft KPro, developed as an improvement over previous versions (II and III). It is made of polytetrafluoroethylene (PTFE), a porous opaque skirt, and polyvinylpyrrolidone-coated polydimethylsiloxane optic, which are chemically bonded together. ${ }^{50}$ This implant is similar in design to AlphaCor and is also inserted into the intralamellar space after dissection of the cornea in its mid-depth. The anterior lamella is trephined in the center to accommodate the optic, whereas the skirt is positioned into the periphery. Lens and iris tissue are removed as necessary. A conjunctival flap or, where it is deficient, a buccal mucosal patch is used to cover over the device. In a later stage, usually after 3 months, the covering tissues are excised with scissors to expose the optic. In a series published by Hollick et al six out of seven cases have failed due to exposure of the haptic, and in one case, it was totally extruded. ${ }^{50}$ In addition, all of these patients required further surgical revisions to stabilize the device. The authors conclude that there is no specific advantage of this device over the AlphaCor.

\section{Hard KPros}

PMMA is proven as the material of choice for the optical component of many KPros. Since it is a rigid polymer, the supporting skirt material has to be sufficiently resilient. Further, the bonding between the skirt and optic should be foolproof to withstand the IOP and forces generated through touch, blinking, and ocular movements. Highly porous and bio-integrable softer skirt materials like Dacron, Teflon, and Proplast were not proven to retain well on the eye. ${ }^{26,30,35}$ Perhaps, harder materials should be preferred to make the skirts in the future KPro models. Moreover, rigid PMMA optic can easily be incorporated into the rigid, than the softer, skirts. The highly successful KPro models like Boston type-1 and OOKP are made of harder skirts.

\section{Boston KPro}

In the early 1950s, renewed interest was brewing in PMMA materials following the accidental discovery of their tolerability in pilots' eyes during the Second World War. Thereafter, many KPros were developed using PMMA as the core optical piece and other biomaterials as skirts. ${ }^{16}$ Since 1960 , a pioneering research program began developing a KPro, which eventually resulted in the current model of Boston type-1 KPro (Massachusetts Eye and Ear Infirmary, Boston, MA, USA). The FDA approved this device in 1992. Previously known as the "Dohlman-Doane Keratoprosthesis", the Boston KPro is made of rigid PMMA material in a collar-stud design. There is a type- 2 version with indications from type- 1 device.

\section{Boston type-I KPro}

This is the most commonly used KPro in the world. It is a two-piece device. The front plate has a convex exterior surface and contains the optical stem. The back plate, made of PMMA, is a disk-shaped piece of $8.5 \mathrm{~mm}$ diameter with a central large aperture and 8-16 small diameter peripheral holes. Previous thread design of the stem is modified into a snug fit type. ${ }^{51}$ A titanium ring locks the back plate onto the stem. The latest design, which is also FDA approved for use in both types 1 and 2, has a modified back plate that eliminates the need for a locking ring and is available in both PMMA and titanium materials. ${ }^{52}$ A donor corneal button with a central trephination is sandwiched between the front and back plates, which acts as a carrier of the device. Fenestrations in the back plate allow aqueous to nourish the donor cornea (Figures 3A and 4). ${ }^{53}$ Boston type-1 KPro is available in standardized pseudophakic and customized aphakic models and can also be supplied with a small back plate ( $7 \mathrm{~mm}$ diameter) for pediatric use. The patients should wear a large diameter soft or contour contact lens for an indefinite period (Figure 3B). The contact lens protects the ocular surface from dryness, desiccation, and against corneal melting, ergo, it enhances device retention. Daily administration of vancomycin eye drops with or without broad-spectrum antibiotics like fluoroquinolones not only prevents infections but also defends against endophthalmitis. ${ }^{54}$

\section{Indications for Boston type-I KPro}

The Boston type-1 KPro is generally used as a corneal replacement in cases when corneal grafting is of high risk, and in the cases of corneal opacities with extensive vascularization and repeat graft failures. Typical examples for such conditions include aniridia, certain corneal dystrophies and degenerations, herpetic keratitis, and corneal infections. ${ }^{55}$ 


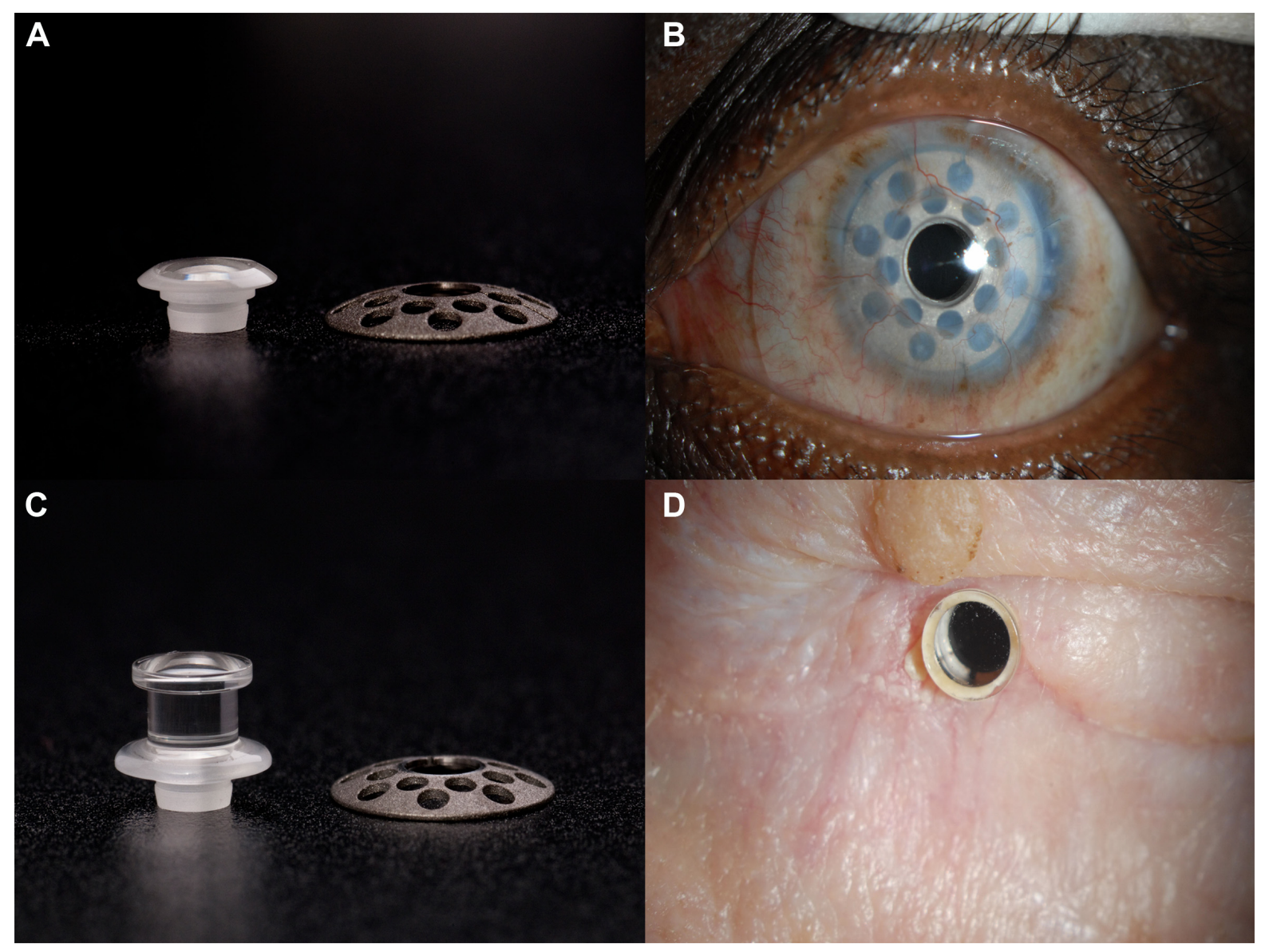

Figure 3 Boston keratoprosthesis.

Notes: (A) Type-I device with front plate (optical stem) and titanium back plate. (B) Type-I device in situ with a bandage contact lens. (C) Type-2 device with extended optical stem and titanium back plate. (D) Type-2 device in situ - projection of the optical stem through the lid. All pictures of this figure are the courtesy of Dr James Chodosh.

An adequate tear secretion and ability to wear a soft contact lens are minimum requirements before considering Boston type-1 KPro. In addition, similar to any KPro procedure, patients should be able to attend regular follow-ups and comply with the postoperative treatment regimens.

\section{Patient follow-up after Boston type-I KPro implantation}

Patients are followed-up frequently in the early postoperative period, as such every week for the first month, then every 2-3 weeks for 3 months, and every 3 months for the first year. Afterward, the follow-ups can be longer and based on the status of the eye. In each visit, visual acuity (VA) is recorded. Periodically, bandage lens must be removed and examined for corneal thinning and loosening of sutures, and a fluorescein dye is instilled to rule out leaks from the "prosthesis-cornea interface" and from the "corneacornea interface". Uniformity of the spread and fit of the bandage lens and its stability with blinks should be noted.
The presence or absence of retroprosthetic membrane (RPM) ought to be checked and may be treated with YAG laser as necessary. IOP can be measured with digital palpation or with a Tonopen at the limbus. The fundus examination is essential, and visual fields should also be tested. Periodically removed bandage contact lenses may be sent for culture and sensitivity tests for microbiological surveillance. Prior to the contact lens replacement, betadine solution may be instilled and retained in the eye for several minutes as a measure to decrease the microbial load on the ocular surface. Any signs of corneal infiltrates or melts should be promptly addressed, which may otherwise result in serious infections and loss of the device, and perhaps eye (Figure 5A). Patients must be warned to return for lens replacement in case they are lost. All patients should receive broad-spectrum antibiotic eye drops like vancomycin or quinolones indefinitely. Topical steroids such as dexamethasone eye drops are usually administered along with the antibiotics for the short term. Their dosage and frequency are titrated and gradually weaned off based 


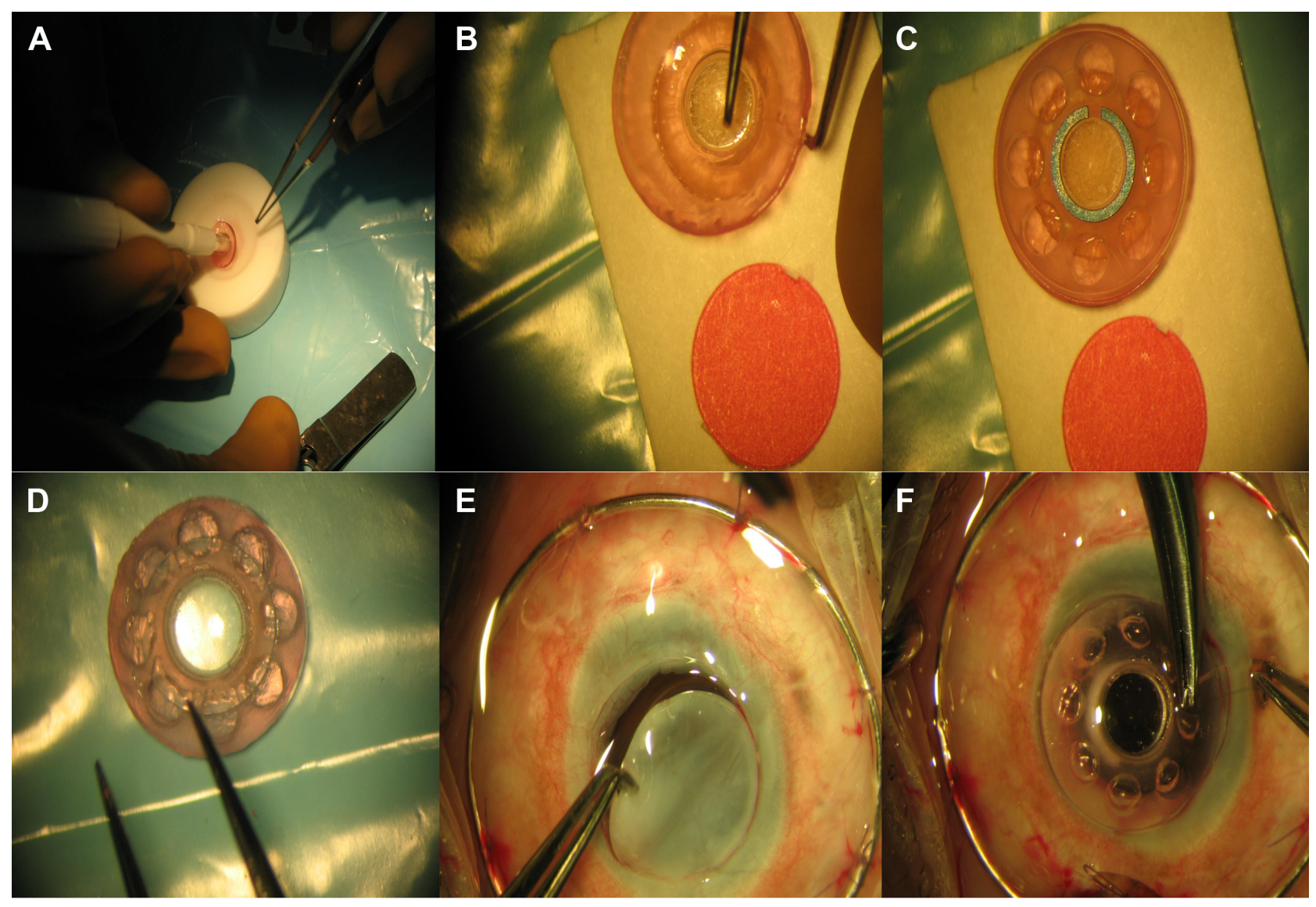

Figure 4 Assembly and implantation of Boston type-I KPro.

Notes: (A) Donor corneal button is trephined to create a central aperture. (B) Corneal graft is placed on the front plate. (C) PMMA back plate covers the graft and titanium ring locks the device. (D) Assembled type-I device ready for implantation (corneal graft is sandwiched between the front and back plates). (E) Host cornea is excised (excised diameter matches outer diameter of the corneal graft). (F) Implantation of the type-I KPro. All pictures of this figure are the courtesy of Dr Geetha lyer.

Abbreviations: KPro, keratoprosthesis; PMMA, polymethylmethacrylate.

on the condition of the eye. Topical anti-glaucoma drops can also be administered in those patients with glaucoma. Preservative-free medications are usually preferred because of the presence of contact lens.

\section{Outcomes of Boston type-I KPro}

In a multicenter descriptive case study, which included 141 Boston type- 1 procedures across 17 centers, Zerbe et al reported a postoperative increment in VA levels up to 20/200 (Snellen equivalent 6/60) in nearly $57 \%$ of patients. ${ }^{56}$ In a case review involving 47 eyes with Boston type-1 KPro, Greiner et al report similar visual results; a $59 \%$ of patients have retained 20/200 VA over a year of follow-up. ${ }^{57}$ Aldave et al published a comparative case study involving various international centers with a cumulative number of 113 procedures, against 110 procedures performed in one of the USA centers. ${ }^{58}$ They noted a preoperative VA level of 20/200 in $2 \%$ of the patients from the international group and in $6 \%$ of the patients from the USA group, whereas postoperatively at 6 months, $70 \%$ of the international patients and $69 \%$ of the USA patients have regained a VA level of 20/200. However, the number of patients maintaining the same level of vision had gradually declined over 2 years to $59 \%$ and $60 \%$, respectively. Interestingly, the percentage of patients with pre- and postoperative VA of less than or equal to light perception was not changed significantly in both the groups (international: $50 \%$ preoperative versus $60 \%$ postoperative; USA group: $9 \%$ preoperative versus $10 \%$ postoperative).

Anatomical retention of the device is excellent in the short term. Zerbe et al report a 95\% retention rate at an average of 8 months of follow-up. ${ }^{56}$ Chew et al report a $100 \%$ retention at 16 months in a cohort of 36 cases, ${ }^{59}$ whereas Aldave et al report an $84 \%$ retention at a 17 -month follow-up for 57 prostheses in 49 patients. ${ }^{60}$ In the comparative series mentioned earlier, Aldave et al identifies a device retention rate of $80 \%$ at a mean follow-up period of 14 months in the international group against a similar retention rate of $80 \%$ at an average of 24 months in the USA group. ${ }^{58}$ Greiner et al 


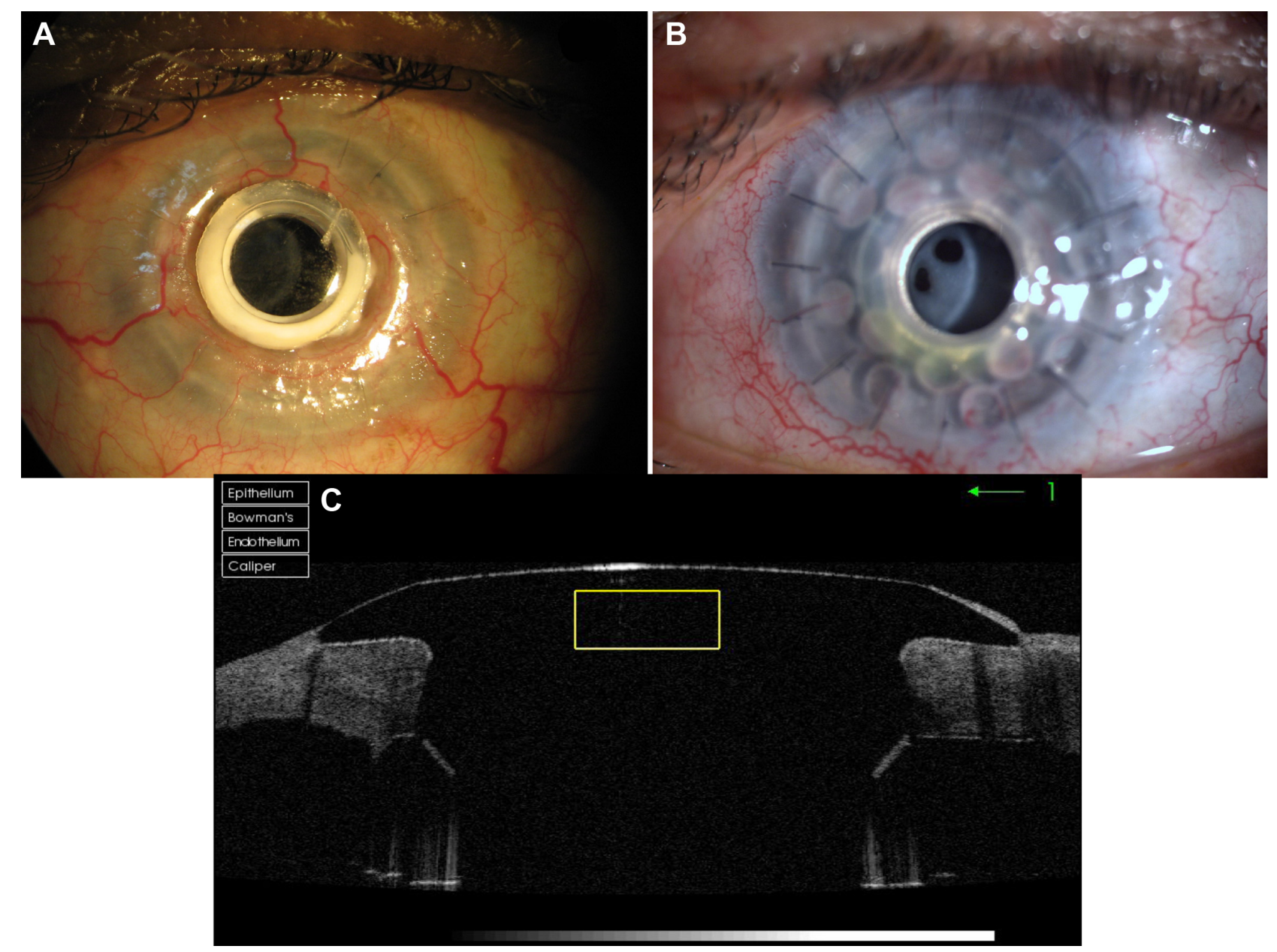

Figure 5 Postoperative course of the Boston type-I KPro.

Notes: (A) Corneal graft melt adjacent to the front plate. (B) Retroprosthetic membrane seen through the optic. (C) OCT scan showing Boston type-I KPro in situ. Pictures (A) and (B) are courtesy of Dr Geetha lyer.

Abbreviations: KPro, keratoprosthesis; OCT, optical coherence tomography.

also reported an $80 \%$ retention rate at a mean follow-up of 34 months. ${ }^{57}$ Bradley et al report an anatomical retention of $81 \%$ for 30 eyes with a mean follow-up of 19 months. ${ }^{61}$ In eyes with successful implants, vision is affected by a number of factors in the postoperative period such as RPM, glaucoma, retinal detachment, endophthalmitis, and stromal melts.

An RPM is a proliferation of the fibrovascular tissue over the internal surface of the device, which can occlude the optical portion leading to visual obstruction (Figure 5B). It is usually treated with YAG laser, but some severe cases will require surgical membranectomy. Stacy et al reviewed the literature and noted an incidence of RPM in the range of $25 \%-65 \%$ with the Boston type-1 implants, of which nearly $45 \%$ required treatment with YAG laser or surgical membranectomy. ${ }^{62}$ They also performed a histological study and hypothesized that RPM is derived from corneal stromal downgrowth from the host side due to gaping of the posterior wound beyond the back plate. In addition, metaplastic lens epithelium and native iris stroma contribute to its development. Magalhães et al in their review, identified a number of risk factors for the development of RPM, which include anterior segment inflammation, previous keratitis, and simultaneous performance of other intraocular surgeries at the time of KPro implantation. ${ }^{63}$

Implantation of a KPro can induce glaucoma and worsen the preexisting glaucoma. Distortion of angle structures, occurrence of RPM, and peripheral anterior synechiae have all been implicated as causes of glaucoma. ${ }^{64}$ According to Banitt, glaucoma is prevalent in up to $36 \%-76 \%$ of Boston KPro patients and de novo glaucoma developed in 2\%-28\% of the patients after the device implantation. ${ }^{65}$ Glaucoma management including its detection, monitoring, and treatment is a significant challenge for KPro surgeons. IOP measurement 
is not possible with corneal applanation methods. Tonopen measurements taken at the limbus and comparisons with the fellow eye have been practiced; subjective estimation of IOP by digital palpation is an alternative, but both are less accurate. Monitoring of visual fields is also constrained due to the smaller size of the optical aperture, which provides limited field of vision. Optic disk photography and imaging with OCT or Heidelberg retinal tomography (HRT) can be useful modalities, but the disk changes can take longer time to develop, and significant field damage could happen in the meantime.

Topical glaucoma medication may be effective in reducing the IOP. Nevertheless, majority of the patients require surgical treatments such as glaucoma drainage device insertions (valved or non-valved) either along with or after the KPro implantation. Li et al studied 45 eyes after Boston KPro implantation and found that 17 eyes needed glaucoma drainage tube insertion. ${ }^{66}$ They also reported a $59 \%$ incidence of conjunctival erosions following glaucoma tube inserts in Boston KPro patients. In the same study, a $60 \%$ of eyes without "glaucoma device-associated conjunctival erosions" retained a VA of 20/200 and only a $25 \%$ of the eyes that suffered erosions could retain a VA of 20/200 at 1-year follow-up. The presence of glaucoma is associated with poor visual prognosis, and development of erosions and subsequent complications, such as hypotony, endophthalmitis, and choroidal and retinal detachments, may adversely affect visual potential of the eye ${ }^{64,66}$ Nevertheless, prompt pre- and postoperative glaucoma management with Boston KPro can improve visual prognosis. ${ }^{64}$ Cyclophotocoagulation can be useful in those who do not respond to drainage tubes. ${ }^{67}$

Robert et al reviewed the literature on endophthalmitis following Boston KPro and found that its incidence ranges from $0 \%$ to $25 \% .{ }^{68}$ Using a pooled data, they estimated the prevalence of endophthalmitis as $5.4 \%$ in the last 10 years with Boston type-1 KPro. The risk of endophthalmitis is generally considered high with inflammatory conditions like SJS, mucous membrane pemphigoid (MMP), and burns. ${ }^{69}$ Although the current standard practice of daily administration of topical vancomycin has reduced the incidence of Gram-positive endophthalmitis, an increased incidence of Gram-negative bacterial and fungal endophthalmitis is observed by some investigators. ${ }^{61,70}$ In cases of endophthalmitis, device explantation followed by vitrectomy and intravitreal injection of broad-spectrum antibiotics is advised in view of the high incidence of posterior segment complications. ${ }^{68}$
Posterior segment complications like retinal detachment have been reported in the range of $3 \%-12 \% .^{56,71}$ Altered eye anatomy and the presence of a limited field of vision through the optic make vitreoretinal surgery a daunting task.

Despite the existence of various problems, there has been a steady increase (more than threefold) in the number of Boston type-1 KPro implantations performed in the USA and rest of the world..$^{72}$ This may be largely due to the increase in device retention rates and awareness of the procedure.

\section{Boston type-2 KPro}

The less popular type- 2 device is similar to type- 1 in design except that it contains a longer optical stem that is intended to project through the lids. It is made for use in severe dry eyes. The ocular surface is divested of the entire forniceal and tarsal conjunctiva, and a notch is created in the upper lid margin to incorporate the extended optical stem, which protrudes beyond the lids. A complete and permanent tarsorrhaphy is performed to conceal the ocular surface along with the device bar the optical stem (Figure 3C and D). Patients with type-2 Boston KPro should also receive prophylaxis with daily topical antibiotic drops like vancomycin and fluoroquinolones. The type-2 KPro is reserved for extremely dry and cicatrizing ocular surface diseases like SJS, MMP, and chemical burns.

Pujari et al in a study involving 29 Boston type-2 KPro implants in 26 patients, reported that $57 \%$ of the patients retained a vision of 20/200 at 1 year. ${ }^{73}$ There was a high prevalence $(70 \%)$ of preexisting glaucoma in their cohort. In the same series, incidence of RPM was 50\%, retinal detachment was $28 \%$, and endophthalmitis occurred in one case. Extrusions of the device were observed in $42 \%$ of the patients.

\section{Recent advances in Boston KPro}

According to Sivaraman et al, development of a thick RPM following Boston KPro is linked to the increased risk of corneal melts and subsequent KPro extrusions by impeding the aqueous flow, thereby decreasing corneal nourishment. ${ }^{74}$ Todani et al suggest that titanium back plates are associated with reduced incidence of RPM formation than PMMA back plates. ${ }^{75} \mathrm{Cru}-$ zat et al suggest that large titanium back plates of $9.5 \mathrm{~mm}$ diameter clamp the graft-host junction more effectively than the $8.5 \mathrm{~mm}$ diameter back plates and may reduce RPM formation. ${ }^{76}$

Anterior segment OCT imaging is a useful adjunct in KPro monitoring (Figure 5C). Pre- and postop imaging can identify patients at risk of developing synechial angle closure. It is also useful in characterization and monitoring of peripheral anterior synechiae and status of the angle. ${ }^{77}$ 
The high-resolution spectral domain OCT may also enable to study complications to the finer details such as RPM, epithelial downgrowth, stromal necrosis, and corneal thinning. ${ }^{78}$ Perhaps, OCT study of preoperative and periprosthetic anatomy could lead to refinements of the design and techniques of Boston KPro device.

Collagen-cross-linked corneas of rabbits with ultravioletA/riboflavin method are shown to be resistant to enzymatic degradation. It has been suggested that cross-linking of donor carrier corneal material could decrease the occurrence of keratolysis and device extrusion. ${ }^{79}$ Frozen donor materials and glycerin-preserved corneas have been suggested as carrier materials for Boston KPro. ${ }^{80}$

Frequent change of the contact lens and periodic administration of antifungal drops and, may be betadine instillation could minimize the risk of fungal and Gram-negative bacterial infections. ${ }^{81}$

Todani et al implanted a ring-shaped wireless IOP transducer (gold microchip and micro-coil antenna encapsulated in a silicone rubber) into the eyes of rabbits after extracapsular lens extraction. ${ }^{82}$ In their study, the device was well tolerated, and the measurements were reproducible and stayed in concordance with manometry. In a subsequent trial by the same group on a patient with open-angle glaucoma, the device was found to be well tolerated over a follow-up of 76 weeks. The IOP measurements were also observed to be in concordance with Goldmann applanation tonometry. ${ }^{83}$ This device may have application in the management of glaucoma associated with KPros.

\section{Osteo-odonto-keratoprosthesis (OOKP)}

Scores of porous and nonbiological keratoprosthesis skirt materials were used, for example, Teflon (Cardona), ${ }^{23}$ Proplast (Girard), ${ }^{24}$ ceramic (Polack), ${ }^{25}$ Dacron (Pintucci et al), ${ }^{28}$ expanded PTFE (Legeais et al), ${ }^{31}$ hydrogel (AlphaCor), ${ }^{47}$ and hydroxy-apatite (León et al) ${ }^{84}$ Integration of these materials with the ocular tissues and longevity of the KPros was not impressive. ${ }^{30,35}$ Consequently, some investigators have tried to utilize autologous tissues for better integration of the KPro. Tooth root-alveolar bone complex (Strampelli-OOKP), ${ }^{85}$ cartilage (Casey), ${ }^{86}$ and tibial bone (Temprano) ${ }^{87}$ were used as the biological alternatives for the KPro skirt. Cartilage is out of use now; however, tibial bone is used as an alternative in edentulous patients when OOKP is not possible.

\section{OOKP surgical technique}

OOKP surgery was invented by Strampelli and subsequently improved by Falcinelli et al ${ }^{85,88}$ Details of the modified OOKP surgical technique are fully described in "Rome-Vienna Protocol". ${ }^{38}$ The basic principle of OOKP involves bypassing the ocular surface with a patch of buccal mucous membrane (BMM) and replacement of the anterior segment structures with an osteo-odonto-acrylic lamina. The mucous membrane can withstand dry conditions of the environment and quickly regenerate after restoration of the moisture, and also survive over inflammation to some extent. The alveo-dental lamina integrates well with the ocular tissues and can retain for a number of decades in successful cases. ${ }^{89}$

OOKP surgery is a complex multi-stage procedure performed by experienced oral and ophthalmic surgeons. Normally, the patients are started on antiseptic and antifungal mouthwashes a day before the surgery. The first stage, which may also be performed in one or two sequential substages, involves laying of the buccal mucosa onto the prepared ocular surface and implantation of the alveo-dental-acrylic lamina into the submuscular pocket (Figure 6). The ocular surface preparation involves removal of the keratinized scar tissues and corneal epithelium and retraction of the conjunctiva to the fornices. A patch of buccal mucosa is excised and grafted to the prepared ocular surface bed. It is also anchored to the four recti muscles for vascular investment. Generally, in the same sitting, a single rooted healthy tooth - typically a canine - with a piece of surrounding mandible is harvested. The tooth-bone plate is trimmed, the crown is excised, and a PMMA optical cylinder is inserted through the central aperture in this plate (Figure 7). The axial length of the eye and surface area of the dentine determine the power and size of the optical cylinder. Thus prepared "osteo-odonto-acrylic lamina" is implanted into the subcutaneous pouch of the contralateral lower lid (Figure 8A and B). The laminar preparation may also be performed at a later stage if the viability of mucous membrane is questionable. In edentulous patients, autografts from tibial bones or allograft teeth from related or unrelated living donors can be considered. . $^{38,40,87}$

The second stage is usually performed after 3 or 4 months to ensure naturalization of the oral mucosa over the ocular surface and to promote vascular ingrowth into the osteoodonto-acrylic lamina (Figure $8 \mathrm{C}$ ). During this stage, the lamina is explanted from the lower lid and examined for erosions, bulk, and stability of the cylinder. It is rare to not be able to proceed further because of an inadequate or unstable OOKP lamina. By trimming off the excess connective tissue from the lamina, the dentine surface is exposed (Figure 9A and B). The mucous membrane is dissected from the sclera on the superior bulbar portion and reflected into the lower fornix to expose the cornea (Figure 9C). An appropriate 


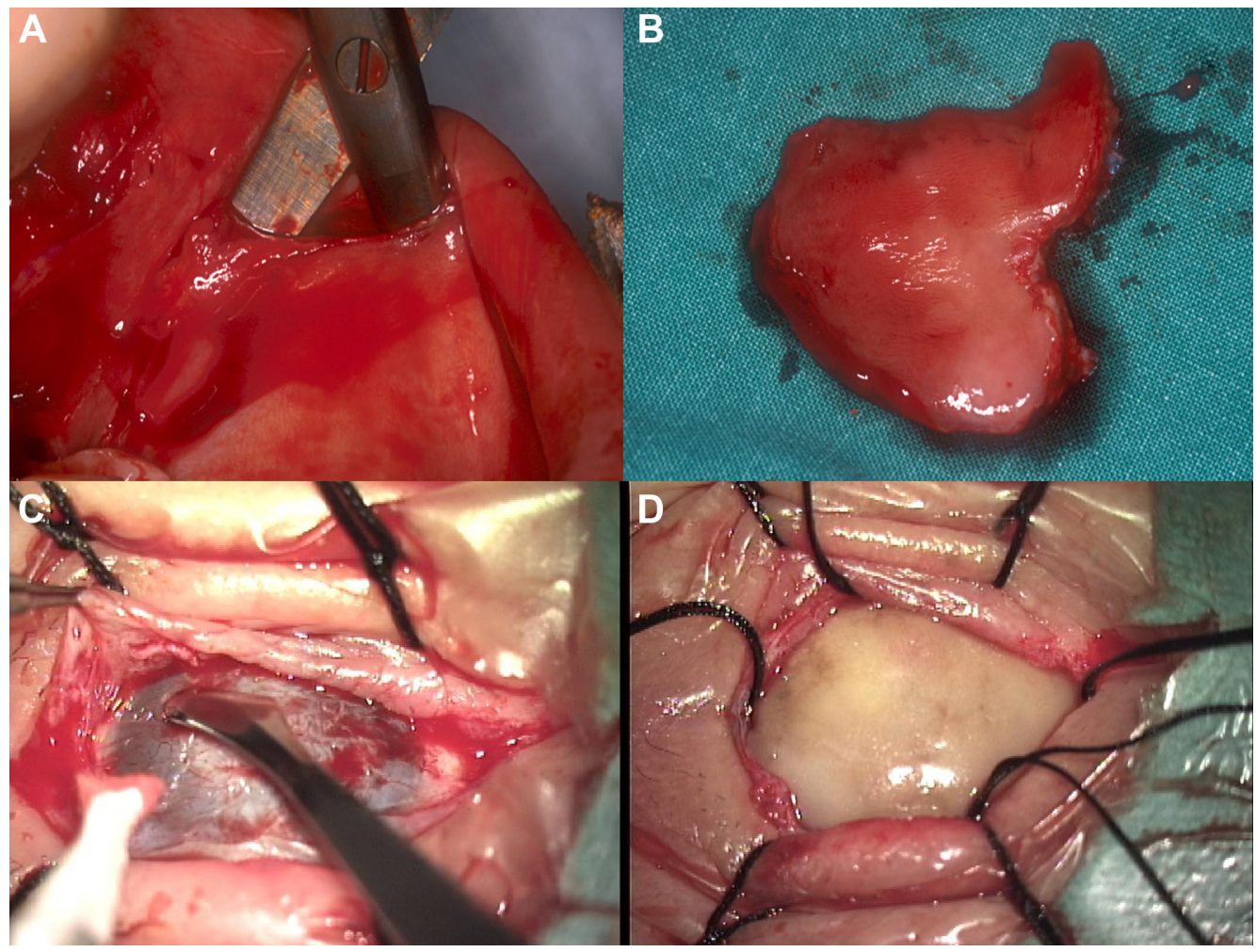

Figure 6 OOKP stage I - buccal mucosal graft preparation and transplantation.

Notes: (A) Excision of the BMM. (B) Excised mucosal tissue-fat and muscle are trimmed off. (C) Ocular surface preparation (sclera is bared and corneal epithelium debrided). (D) Mucosal graft transplantation on to the ocular surface.

Abbreviations: OOKP, osteo-odonto-keratoprosthesis; BMM, buccal mucous membrane.

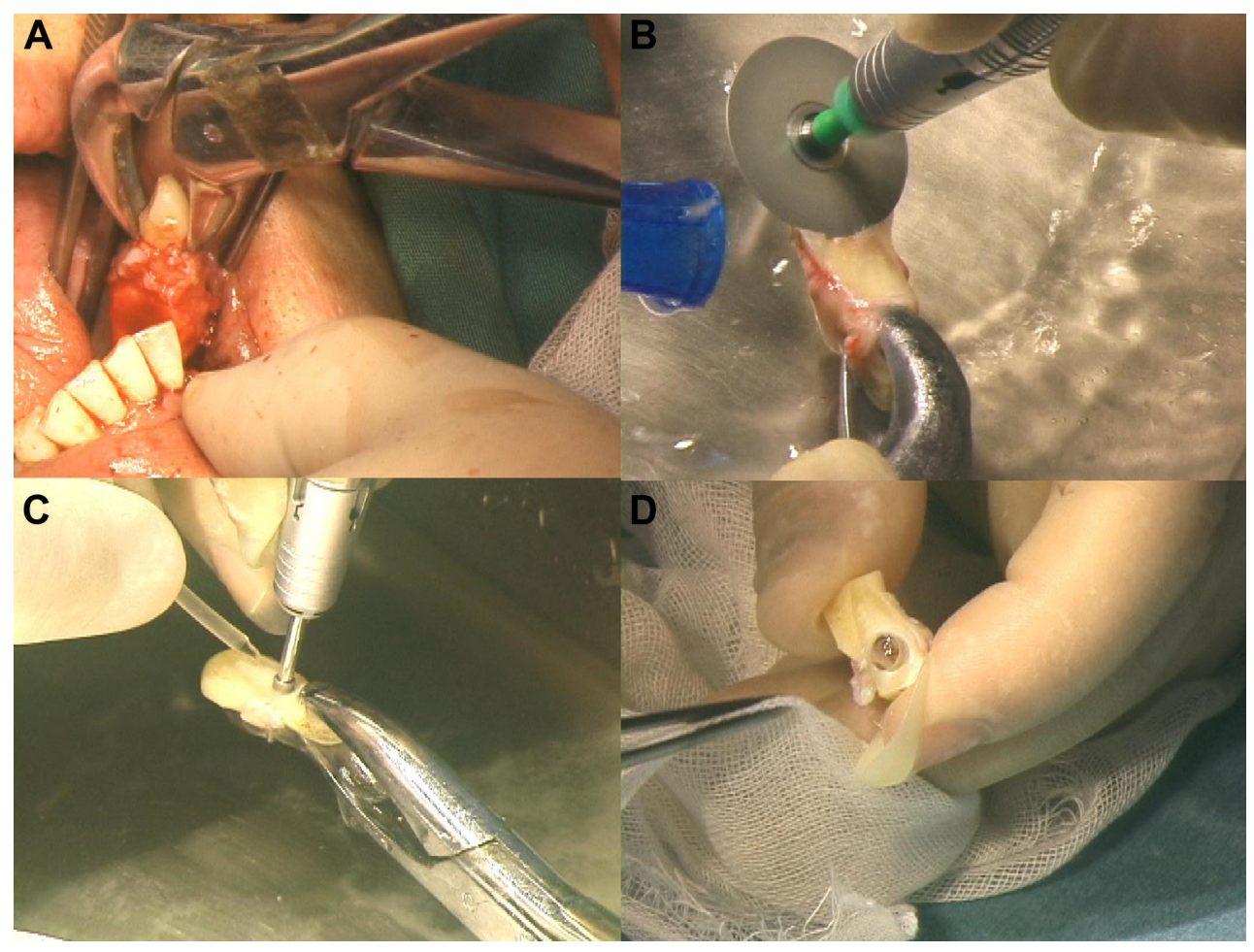

Figure 7 OOKP stage I - tooth extraction and preparation of the OOAL lamina.

Notes: (A) Extraction of the tooth with a piece of mandible. (B) Preparation of the tooth (dentine is exposed on one surface, while the alveo-dental ligament is preserved). (C) A central hole is drilled perpendicular to the lamina. (D) A PMMA cylinder is inserted into the lamina.

Abbreviations: OOKP, osteo-odonto-keratoprosthesis; OOAL, alveo-dento-acrylic; PMMA, polymethylmethacrylate. 


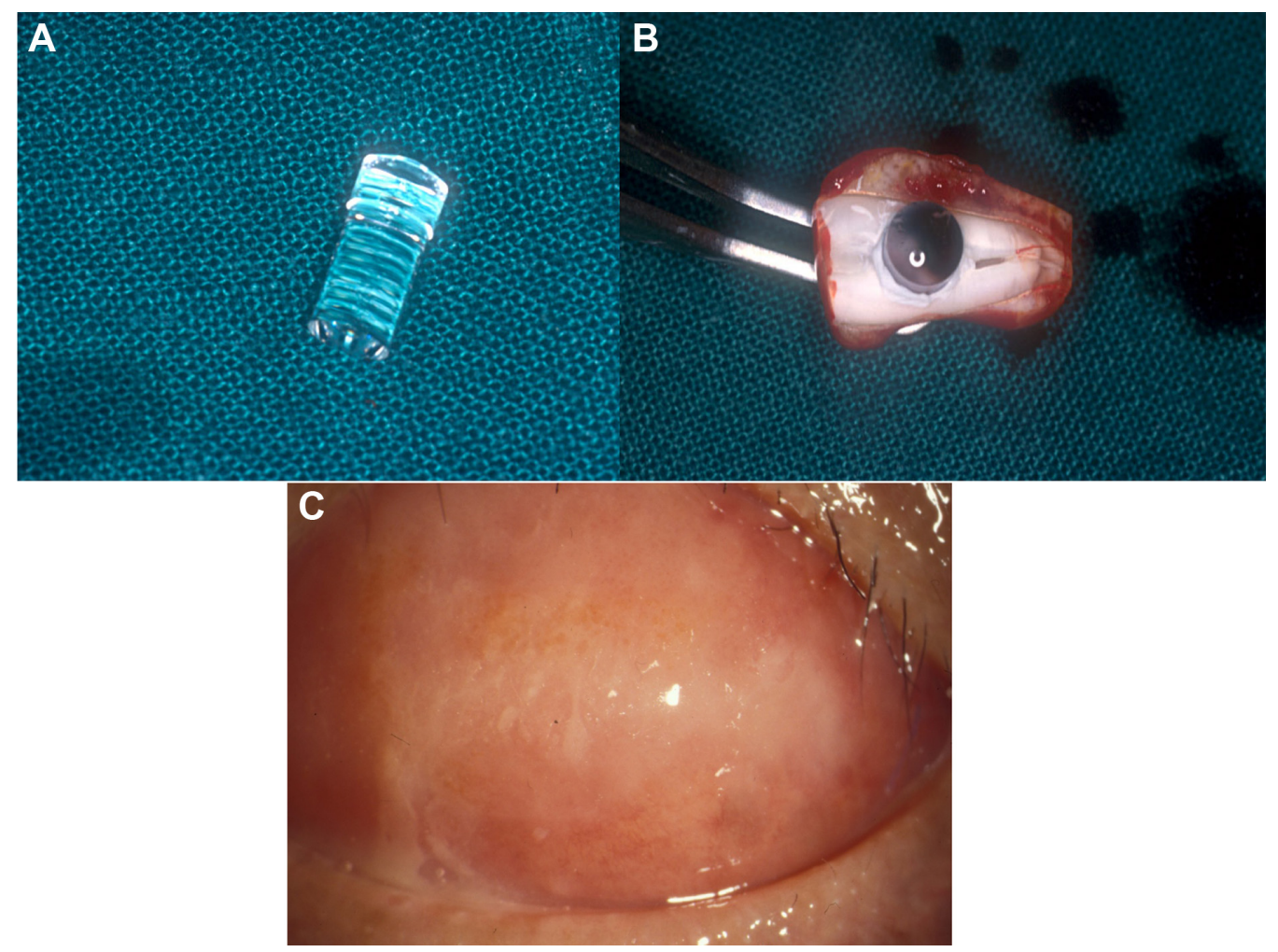

Figure 8 OOKP lamina and postoperative course after stage $\mathrm{I}$.

Notes: (A) PMMA optical cylinder - the wide part sits on the dentine surface. (B) Prepared OOAL lamina - rim of acrylic cement can be seen around the optic. (C) A healthy mucous membrane I month after the stage I.

Abbreviations: OOKP, osteo-odonto-keratoprosthesis; PMMA, polymethylmethacrylate; OOAL, alveo-dento-acrylic.

diameter Flieringa ring is fastened to support the sclera. Any left-over corneal epithelium is removed, and the cornea is trephined in the center to create a 3-4 mm diameter aperture, which is extended by two opposite slit incisions (Figure 9D). The crystalline lens is removed by cryoextraction (Figure 9E); if pseudophakic, intraocular lens is also removed with the bag in toto, redundant iris is removed from its root, and core vitrectomy is performed (Figure 9F). The lamina is implanted by insertion of the optical cylinder through the central corneal hole (having its dentine side sitting on the cornea). The lamina is anchored to the sclera with vicryl (6-0) sutures (Figure 9G). Cylinder positioning is assessed with an indirect ophthalmoscopic examination, and the sutures are adjusted accordingly to achieve centrality. The whole lamina should be tightly secured. The BMM is closed with interrupted absorbable sutures. An opening is made in the center of BMM, through which the optical cylinder projects up to $2 \mathrm{~mm}$ (Figure 9H). ${ }^{38,40}$

Aseptic precautions must be observed while handling the tissues. The mucous membranes should always be kept moist. The osteo-odonto lamina is to be stored in the heparinized blood during the transit. Prophylactic broad-spectrum antibiotics, intravenous acetazolamide, and mannitol are given during the operations to reduce the risk of subchoroidal hemorrhage. ${ }^{40}$

\section{Indications and contraindications for OOKP}

Full details of the OOKP surgery including indications, investigations, patient assessment, and postoperative care can be accessed from Liu C et al. ${ }^{40}$ OOKP is indicated for eyes with defective lids and blinking, surface keratinization, and severe inflammatory conditions of the ocular surface such as: SJS, MMP, chemical and thermal burns, Lyell syndrome, trachoma, GVHD, and Sjögren's syndrome ${ }^{88}$ OOKP is not suitable for children due to the high bone turnover. It is contraindicated in phthisis bulbi and eyes without light perception. ${ }^{38,40}$

\section{Patient assessment for OOKP}

The patient assessment process in our institution (Sussex Eye Hospital, Brighton, UK) is similar to that mentioned under the "General considerations for KPro surgery" section. A multidisciplinary team comprising of ophthalmologists, 

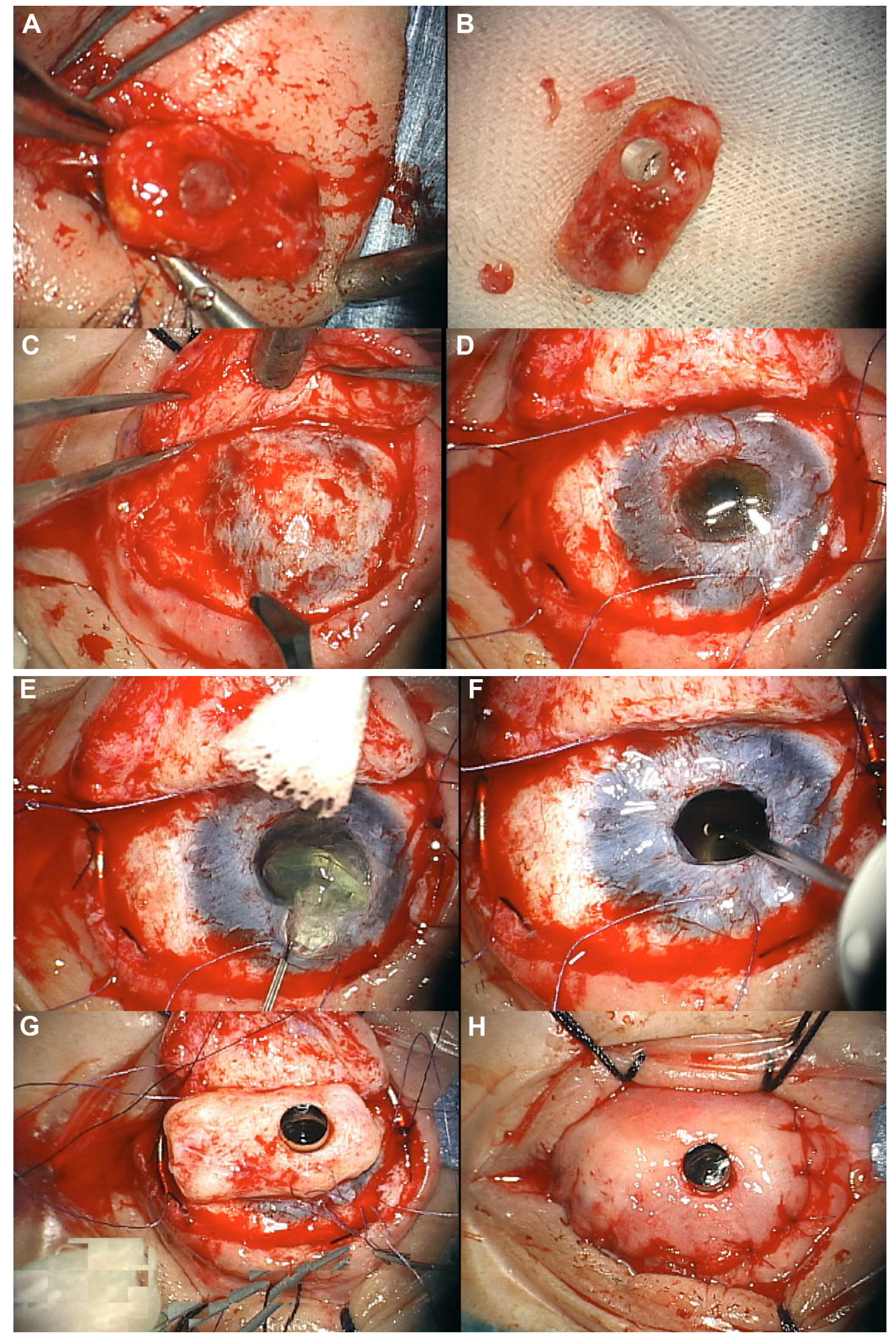

Figure 9 OOKP stage 2 - retrieval of the lamina and implantation into the eye.

Notes: (A) Lamina is recovered from the subcutaneous pocket. (B) Connective tissue is removed from the lamina to expose the dentine side. (C) Buccal mucosa from the eye is reflected to expose the cornea. (D) Central corneal button is excised. (E) Lens extraction (IOL is also removed when present). (F) Open sky core vitrectomy is performed. (G) Lamina is implanted into the eye with wide portion of the optical stem passing through the cornea. (H) Mucosal membrane is replaced over the lamina. Through a central opening in the BMM, the optic projects beyond I $\mathrm{mm}$.

Abbreviations: OOKP, osteo-odonto-keratoprosthesis; BMM, buccal mucous membrane; IOL, intraocular lens. 
oral surgeons, anesthetists, nurses, and a clinical psychologist assesses patients referred for KPro surgery to our institute. The aim of the assessment is to establish the suitability of the patients for OOKP surgery, to identify the risk factors that could influence the surgical outcomes, and to provide information to the patient and family. The ophthalmologist is the initial point of patient assessment. During this consultation, the underlying pathology is determined, and the status of the eyes is evaluated as mentioned earlier. The eye for the proposed surgery is chosen based on the potential for success and patient's preference. In the same visit, a short examination of the oral cavity and dentition is performed, general health is evaluated, and patients are subsequently referred to the oral surgeon and clinical psychologist if they are found suitable for OOKP surgery.

The oral surgeon assesses and selects the teeth based on clinical and radiological examination of the patient's oral cavity. An orthopantomogram is usually performed. Some patients have poor dental cleanliness and oral health because of the co-existing disease of the oral cavity, for example, SJS. These patients are advised to improve oral hygiene and stop smoking if applicable. For edentulous patients, related or unrelated tooth donors may be considered and screened as required.

The clinical psychologist's assessment is pivotal in our practice. Patients are thoroughly investigated for their psychological and general health by the multidisciplinary team. Patients are educated about the OOKP surgery and provided with printed leaflets. Surgery is generally offered to patients with bilateral poor vision. The eye with a good visual potential is usually selected. Subsequent visits are planned to help the patients to arrive at a decision. The family members, patient carers, and tooth donors (if any) are also consulted. Once a decision is made for the surgery, they are referred to the anesthetist for assessment.

\section{Follow-up after the OOKP Surgery}

After the OOKP procedure, patient's follow-up is lifelong. After each stage of the procedure, patients are usually seen every week in the first month and every month for the next 3 months, and the further follow-up intervals are prolonged based on their progress. At every procedure, broad-spectrum antibiotics, oral acetazolamide, and steroids are given for a week, and patients are nursed in the hospital for a minimum of 1 week. Patients should be warned before the stage 1 OOKP surgery that their vision could be further reduced because of the mucosal overlay on the cornea. In the early weeks after the stage 1 , they are closely monitored for mucosal graft viability on the eye. They receive a broad-spectrum antibiotic eye ointment for three to four times a day to lubricate the mucosa. A conformer is also placed over the mucous membrane to prevent formation of adhesion bands. Patients are encouraged to do wide mouth opening exercises, and massage the cheek area externally over the site of mucosal harvestation to prevent stricture formation.

IOP measurement by tactile method and B-scan ultrasonography must be performed. Any raise in IOP should be treated with oral acetazolamide or a glaucoma tube.

In the later weeks, BMM over the ocular surface is inspected for ulcers, thinning, and infections. Any pathology should be promptly attended. If patients develop mucosal complications and require further treatments, it could delay undertaking of the stage 2. Leaving the lamina for longer than 3 months in the submuscular pouch can result in laminar resorption even before proceeding to the stage 2 . It could necessitate a new lamina preparation, consequently leading to repetition of stage 1 . Therefore, every effort should be made to promptly address mucosal complications and not to prolong the waiting time for stage 2 .

Inspection of the lamina implantation site, on the contralateral lower lid, is not to be forgotten. Lamina should be palpated at each visit; the presence of tenderness or swelling over the laminar site may denote infection. If infection develops, it should be treated with broad-spectrum systemic antibiotics and may require laminar removal. An infected lamina may not be suitable for implantation into the eye because of the risk of endophthalmitis or its resorption.

In subsequent visits after the second stage, VA, mucosal health, laminar bulk, optic stability, and IOP are checked (Figure 10A). Fundus examination may not be possible in the early days after the stage 2 due to the presence of hemorrhage and air in the vitreous. Three or four months following the second stage, patients are advised to visit their opticians for refractive assessment and spectacle correction. We expect a change in the refractive status by that period due to loosening of the sutures and re-expansion of the globe. A rapid or a large change in refractive error occurring after this period may be due to migration of the optic or lamina as a result of laminar resorption. Periodic computerized tomography (CT) scanning of the lamina is performed to monitor its dimensions and volume.

Allograft patients are normally started on oral cyclosporine in the immediate postoperative period; however, the optimal dosage and duration of such treatment are unknown. Cyclosporine serum trough levels between $100 \mathrm{ng} / \mathrm{mL}$ and $200 \mathrm{ng} / \mathrm{mL}$ are maintained for allograft patients in our 


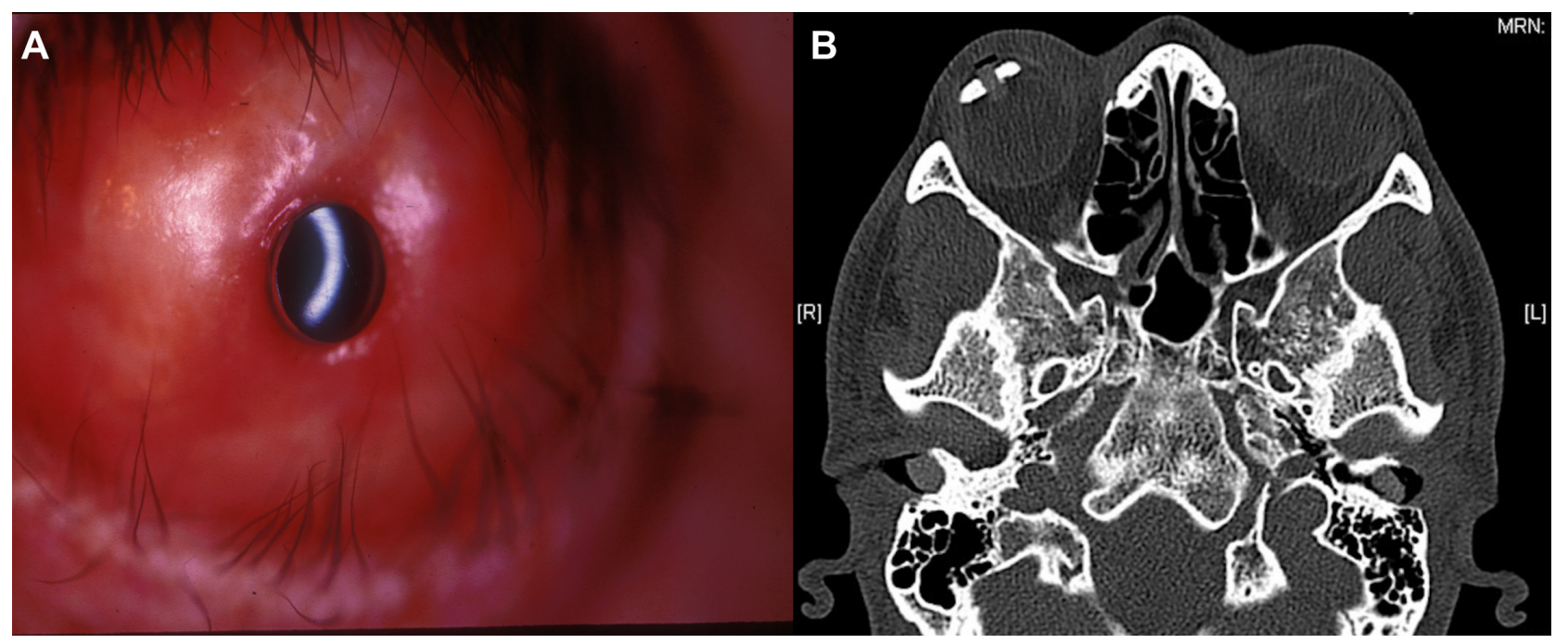

Figure 10 Postoperative course after stage 2.

Notes: (A) A healthy OOKP eye after a successful surgery. (B) CT scan image showing an intact lamina in the OOKP eye.

Abbreviations: OOKP, osteo-odonto-keratoprosthesis; CT, computerized tomography.

practice. We also prescribe oral biphosphonates for patients with early laminar resorption and allografts in the anticipation of protection against laminar loss. There is insufficient follow-up data to verify the effect of this strategy. Patients being maintained on cyclosporine, oral steroids, and acetazolamide over the long term should be monitored for their side effects in liaison with their local physicians. ${ }^{40}$

\section{Outcomes of OOKP}

Long-term anatomical retention of the OOKP is excellent across the major published studies. Liu et al reported an $81 \%$ probability of retaining laminar autografts over 5 years from a cohort of 36 cases,${ }^{90}$ whereas the 5 -year probability of retaining allografts was nil from his cohort. The median survival of OOKP allografts was 30 months in their series. Marchi et al reported a $98 \%$ retention of the lamina in 85 patients over a 20 -year follow-up. ${ }^{91}$ Falcinelli reported an $80 \%$ probability of laminar retention in 224 cases over a follow-up of 18 years. ${ }^{92}$ From Michael et al's report, 10-year anatomical survival of 145 OOKP and 82 tibial KPro implants was $66 \%$ and $47 \%$, respectively. ${ }^{93}$ Hille et al reported $100 \%$ retention of OOKP laminae in a 5 -year follow-up. ${ }^{94}$ Iyer et al reported a $96 \%$ anatomical success of OOKP laminae in 50 cases with a mean follow-up of 15 months. ${ }^{95}$

A major cause for anatomical failure of the OOKP is laminar resorption. Liu et al noted that laminar resorption occurred in $19 \%$ of their cases..$^{90}$ Iyer et al reported laminar resorption in $22 \%$ of patients. ${ }^{95}$ De La Paz et al reported a laminar extrusion of $28 \% .{ }^{96}$ Resorption results in decreased thickness and defects in the lamina. When resorption involves the central aperture of the lamina, the optic cylinder can become loose and unstable, which can eventually result in aqueous leak, altered refraction, tilting of the lamina with change of visual axis, and endophthalmitis leading to catastrophic visual loss. Clinical detection of laminar resorption can be difficult until late sequelae such as laminar tilt and loosened cylinder have developed. In some cases, endophthalmitis may be the first clinical manifestation of laminar resorption.

Serial imaging of the lamina and comparison of its dimensions with $\mathrm{CT}$ or electron beam tomography can detect resorption early (Figure 10B). ${ }^{97}$ Stoiber et al proposed that evaluation of laminar linear dimensions on serial CT reconstruct images could identify resorption. ${ }^{98}$ However, their method is not entirely objective and there is an element of operator-induced subjectivity and data may not be reproducible. In a retrospective study, Sipkova et al applied an automated algorithm called "Advanced Lung Analysis Software" on UK national cohort of OOKP patients and analyzed laminar volumes objectively from the serial multidimensional CT images. ${ }^{99}$ This method is reproducible and identified resorption earlier than clinical examination in $60 \%$ of the patients. Early detection of resorption could help the clinicians to monitor the rate of bone loss and enables to intervene at the correct opportunity. Risk factors for laminar resorption include allografts, young age (contraindicated in less than 17 years), tibial lamina, persistent inflammation, and perhaps smoking and use of steroids. ${ }^{38,40,87,89,90,93,97}$ Patients with these risk factors should be watched more closely.

In a systematic review, Tan et al conclude that across the published studies, $52 \%$ of patients achieved a VA better than 
6/18 after OOKP and tibial lamina implantations. ${ }^{89}$ Liu et al gave an account of visual results from the UK and stated that out of a total of 36 patients, $78 \%$ have achieved a VA of $6 / 60$ or better and $53 \%$ achieved $6 / 12$ or better. ${ }^{90}$

Tan et al and Hille et al reported intraoperative vitreous hemorrhage in nearly $50 \%$ of their cases. ${ }^{89,94}$ In the early postoperative period, the main causes for slow regain of VA are the presence of air bubble and hemorrhage in the vitreous. In our experience, some patients could take three to six months to develop cortical adaptation to vision. Elderly and those with a longstanding blindness before the OOKP operation should be prepared for delayed visual recovery.

In anatomically successful OOKP eyes, the main cause of visual loss is glaucoma. Glaucoma occurrence varies across the studies as identified by Tan et al in their review (Hille - 16\%, De La Paz - 17\%, Iyer - 20\%, Marchi - 33\%, Tan - 34\%, Liu - 47\%). ${ }^{89}$ In Falcinelli's series, incidence of de novo (true postoperative) glaucoma was $10 \% .{ }^{92}$ In a recent retrospective review of our unpublished data from the UK national cohort of OOKP patients involving 47 cases, we identified that $40 \%$ had pre-existing glaucoma, and $15 \%$ developed de novo glaucoma following the OOKP operation. Topical treatment with eye drops has only a limited role in treating OOKP eyes. In our experience, oral acetazolamide, sublingual administration of timolol eye drops, and oral beta-blockers have some beneficial effect on lowering the IOP. Surgical treatment is usually with the drainage tubes or ciliary body ablation using either transscleral or endoscopic lasers. Insertion of glaucoma tubes is normally not performed at the time of OOKP surgery to avoid hypotony, which may jeopardize laminar stability before it integrates with the sclera. Baerveldt implants may be considered for simultaneous surgery by temporarily occluding the lumen with sutures. However, insertion of any drainage device is fraught with difficulties due to lack of clear anatomical landmarks. In addition, the effect of the device is compromised by thick BMM that impedes episcleral drainage.

Detection and monitoring of glaucoma is difficult in OOKP eyes similar to other KPros. Objective measurement of IOP in OOKP eyes with instruments is not possible. Therefore, digital IOP measurement by the clinician, disk assessment clinically and by serial disk imaging with photography, OCT, or HRT, and periodic visual field testing are useful in glaucoma assessment. ${ }^{100}$ In a study by Falcinelli et al the pattern electroretinogram amplitudes and contrast sensitivities are reduced in OOKP patients. ${ }^{101}$ Visual-evoked potentials are significantly reduced in glaucoma patients with OOKP compared to normal OOKP subjects. ${ }^{101}$ In our experience, some patients report eye pain and frontal headache associated with raised IOP that is relieved after starting or increasing the uptake of oral acetazolamide. Complaints of reduced vision and eye pain should raise the suspicion of increased IOP in OOKP patients, particularly in de novo glaucoma cases.

RPM developed in 1\%-7\% in studies with large number of patients, ${ }^{92,96}$ compared to $17 \%-20 \%$ in smaller studies. ${ }^{89,90}$ RPM generally requires YAG laser treatment similar to capsulotomy. In resistant cases, surgical membranectomy may be indicated. In severe cases, an exchange of lamina may be necessary.

Hughes et al reported a $23 \%$ incidence of vitreoretinal complications from the UK cohort of 35 patients that included vitreous hemorrhage (three cases), retinal detachments (three cases), and sequelae of endophthalmitis (three cases). ${ }^{102}$ Pars plana vitrectomy was performed in these patients through a temporary KPro using binocular indirect viewing system or under endoscopic guidance. ${ }^{102}$ Tan et al found a variable incidence of retinal detachment, 3\%-26\%, across the studies and a $6 \%$ in their own series. ${ }^{89,103}$ Managing vitreoretinal complications can be challenging because of the limited view and glare through the optic, and hence requires higher surgical expertise.

Oculoplastic complications account for a majority of the surgical procedures performed on OOKP eyes. Among the reported studies, mucosal complications were lowest at $8 \%$ in Falcinelli's study and highest in Hille et al's study at 48\% ${ }^{89,92,94}$ Liu et al and Tan et al reported mucosal complications in $28 \%$ and $25 \%$ of their cohort of cases, respectively. ${ }^{89,90}$ The type of cases and the risk factors vary among these studies that may explain the differences in mucosal complications. Ulceration of the mucous membrane usually occurs due to inadequate vascularization of the graft on the ocular surface. These problems are usually treated with a bucket-handle flap, tarsal pedicle flap, or a new mucosal patch graft. Mucosal membrane overgrowth was reported in the literature by Iyer et $\mathrm{al}^{95}$ in $2 \%$, by Liu et $\mathrm{al}^{90}$ in $33 \%$, and by Tan et $\mathrm{al}^{89}$ in $22 \%$ of the cases. Mucosal overgrowth is normally treated by surgical excision. In recurrent cases, mucosal trimming and mitomycin-C application around the optical cylinder prevented mucosal regrowth in our experience. ${ }^{104}$ Intraoperative oral complications were reported in nearly $1 \%-3 \%$ of cases in Rome series. ${ }^{92}$

The incidence of endophthalmitis was lowest in Falcinelli's series at $2 \% .{ }^{92}$ Liu et al reported an $8 \%$ incidence of endophthalmitis in their cohort. ${ }^{90}$ However, their series 
contained OOKP allografts. Endophthalmitis risk is high in the post-laminar implantation period either following a surgical procedure or because of laminar resorption. Risk factors for endophthalmitis in an OOKP eye include laminar resorption with loosening of the optic, indwelling glaucoma tubes, and noncompliance with antibiotic ointment. Resorption can lead to expansion of the laminar foramina that creates a potential conduit for microbial entry into the globe. The risk of endophthalmitis may be low in OOKP eyes since there is no use of contact lens or corneal melt. Patients with acute endophthalmitis usually present with pain and visual loss. Its treatment may require laminar explantation, vitrectomy, and injection of intravitreal antibiotics. The prognosis is poor. ${ }^{97}$

\section{Seoul (S-KPro)}

Latest iteration of this KPro, developed by Kim et al includes an umbrella-shaped skirt made of polyurethane and nonwoven polypropylene, whereas the primary model of this device included an expanded PTFE skirt. ${ }^{105}$ The optic is made of PMMA, which also harbors two polypropylene haptics. The skirt is fixed into the intralamellar corneal pocket, while the haptics are fixed to the sclera by ab interno insertion. Kim et al have published the outcomes of their device in nine eyes with an average follow-up of 63 months. All of the cases had exposure of the skirt, and device exchange was needed in four eyes. ${ }^{105}$

A

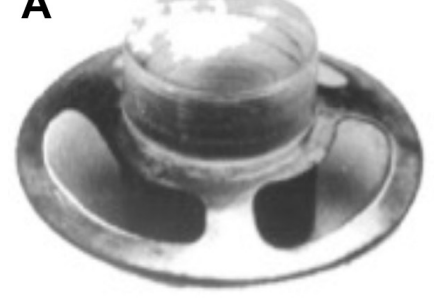

E

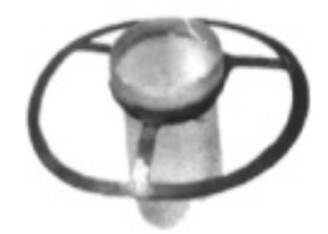

B

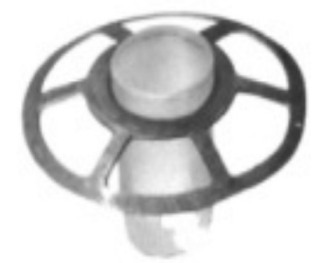

$\mathbf{F}$

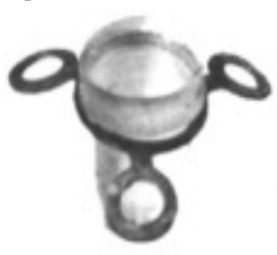

\section{KPros with titanium skirt}

Titanium has been in use for biomedical applications for a long time. Its use in keratoprosthetic applications dates back to five decades. Apart from the recent Boston KPro model, which has a titanium back plate, KPros based on titanium metallic skirts have been in use from a long time in Russia, Ukraine, and the People's Republic of China.

\section{KPro at Filatov Institute, Ukraine}

Filatov institute in Ukraine has been involved in the development of a KPro since 1966. ${ }^{106}$ They used titanium flanges to support the PMMA optic, and thus a range of designs were developed at their center (Figure 11). The newest variety of their device, "Iakymenko-Golubenko model", consists of a central titanium ring, which bears the threaded optic, and three small ring-shaped flanges attached on its outside. This KPro is implanted in two stages. In the first stage, the device is fixed ab interno; the flanges are inserted into patient's corneal lamellae and optic into the anterior chamber. The anterior corneal lamella is retained for 3-6 months to cover the optic and establish the device inside the eye. In the second stage, the anterior corneal lamella is excised over the front surface of the optic, which then enables the patient see through. The host cornea is strengthened externally to endure the metallic skirts by the addition of corneal graft or oral mucosal tissue. Intralamellar strengthening is also achieved in some cases by using a donor lamellar corneal 
graft, or dura mater or autologous auricular cartilage. Iakymenko published the results of different models of KPros implanted in 1,060 eyes between the years 1972 and 2010 at their institute. ${ }^{106}$ With his latest design of dismountable threaded optic, as mentioned above, and by strengthening of the host cornea, he projects a steep reduction in corneal melts from $29 \%$ to $3 \%$ and device extrusions from $17 \%$ to $1.5 \%$. The exact duration of follow-up is not clear from his publication. He proposes that his device has a success profile similar to that of Boston type-1 KPro and OOKP and is suitable for dry and wet eyes. A multicenter study with long-term follow-up may establish this interesting prosthesis.

\section{MICOF KPro}

Moscow Eye Microsurgery Complex in Russia (MICOF) KPro, which is used in Beijing, People's Republic of China, is similar to the Ukrainian KPro in material composition and built. ${ }^{107}$ It has a titanium frame of two ear-shaped flanges and a central ring. The ring incorporates a threaded PMMA optic. This device is also implanted in two stages. During the first stage, the titanium frame, with a filling material inside the ring, is implanted into the corneal lamella. In the second stage, the cornea is trephined, filling material is removed, and posterior corneal lamella is trephined inside the central titanium ring. The PMMA optic is screwed inside the ring to reach the anterior chamber. The procedure is completed by a pars plana vitrectomy combined with removal of the lens and iris. Huang et al published the results of 85 cases of MICOF KPro. ${ }^{107}$ Their series contained a large number $(82 \%)$ of patients with chemical and thermal injuries, and the remainders were autoimmune diseases. They projected a device retention rate of $81 \%$ at a mean follow-up of 35 months. They also stated that none of the devices were explanted, but $19 \%$ of the cases required tissue augmentation to retain the implant. There was a high incidence of RPM (46\%) in their cohort. Although authors have used autologous auricular cartilage to strengthen the cornea, use of this device in cases with previous perforations and corneal grafts may be difficult. Likewise, a multicenter study with a longer term follow-up may establish the success of this KPro.

\section{Fyodorov-Zuev KPro}

Another device similar to MICOF KPro, namely FyodorovZuev KPro, is made of two ear-shaped titanium flanges having large holes and a central titanium ring with internal threading that matches with the external threading of the PMMA optical cylinder. This device is first inserted into the lamellae of a donor cornea which is then implanted into the eye akin to a standard penetrating keratoplasty. The lens, iris, and mid-vitreous are also removed in the same sitting. The entire complex may be covered by conjunctival flap or labial mucosa. Ghaffariyeh et al published a series of ten cases using this device with a follow-up ranging between 28 months and 84 months. ${ }^{108}$ The device was retained only in seven cases. Its success was limited by complications like corneal necrosis around the central piece and poor integration leading to aqueous leaks, hypotony, and endophthalmitis.

Essentially, the titanium devices are implanted within the corneal lamellae. These prostheses may find application in cases, where corneal strength is adequate to support the device. Although the authors have adapted to corneal strengthening procedures by adding exogenous or autologous materials, integration and longevity of the device are questionable in conditions such as thin corneas, previous perforations, existing corneal grafts, and recurrent herpetic keratitis. Nevertheless, these devices could be considered as alternatives to OOKP when patients have no suitable teeth.

\section{Conclusion}

The Boston type- 1 and OOKP are the two KPros with a proven record of success among numerous devices that came into human use. They have rapidly gained popularity across the world. Boston type-1 KPro is widely considered as the device of choice to restore vision in "wet-blinking eyes", and OOKP for eyes with dryness and defective lids and blinking.

The Boston KPro has the advantage of easy repeatability in cases of device failure since it is available off the shelf. Yet, it requires a donor cornea as a carrier. OOKP is a complex multi-stage procedure, which is time and resource hungry. In comparison to OOKP, Boston type-1 KPro surgical procedure is technically less challenging but has a less follow-up record. OOKP, with a well-established long-term success, is the "gold standard KPro", against which other KPros should be evaluated.

Edentulism is common in elderly and patients with oral mucosal diseases. Lack of suitable dentition is a limitation for a number of patients in need of OOKP surgery. Ophthalmologists involved in the management of patients who are potential candidates for future OOKP surgery should pay attention to the oral care of the patients, and may perhaps involve dentists early on the course of patient management.

Patient selection is crucial for successful outcome with any KPro surgery. Patients considering a KPro should be able to accept possible complications, further operations, and frequent hospital visits. They should also be able to 
recognize sudden changes in their conditions and present quickly to the hospitals for emergencies. Psychological and social support is of paramount importance in the patient care. The level of available social care and capacity for self-care must be clearly evaluated before offering the KPro procedure. After a prosthesis implantation, patient follow-up is lifelong. Selection of the correct device for the correct patient, with due consideration of the underlying diagnosis and morbidity of the eye, is fundamental for best outcomes. Switching patients from one device to other after an implantation may not be possible.

After a successful KPro implantation, further surgical revisions will be required to retain the prosthesis and to treat complications. Despite these measures, glaucoma can adversely affect the long-term visual outcome. Therefore, a KPro should be considered as a non-permanent restoration of sight at this point of time.

PMMA has largely solved the need for a stable and biocompatible optical component of KPros. Nonetheless, the anchoring skirt material is still an area that requires research. We envisage that with rapid advances in biomaterial science and surgical techniques, and the success of the KPros and their longevity will continue to improve.

\section{Acknowledgments}

We sincerely acknowledge Dr Qureshi Maskati, Dr James Chodosh, Dr Geetha Iyer, and Prof Stanislav Iakymenko for providing us illustrations to include with this article.

We also acknowledge Macmillan publishers for the granting of license to reproduce the illustrations of the AlphaCor KPro.

\section{Disclosure}

The authors report no conflicts of interest in this work.

\section{References}

1. Williams KA, Esterman AJ, Bartlett C, Holland H, Hornsby NB, Coster DJ. How effective is penetrating corneal transplantation? Factors influencing long-term outcome in multivariate analysis. Transplantation. 2006;81:896-901.

2. Price FW Jr, Whitson WE, Marks RG. Progression of visual acuity after penetrating keratoplasty. Ophthalmology. 1991;98(8):1177-1185.

3. Coster DJ, Williams KA. Management of high-risk corneal grafts. Eye. 2003;17:996-1002.

4. Vanathi M, Panda A, Vengayil S, Chaudhuri Z, Dada T. Pediatric keratoplasty. Surv Ophthalmol. 2009;54:245-271.

5. Bachmann B, Taylor RS, Cursiefen C. Corneal neovascularization as a risk factor for graft failure and rejection after keratoplasty: an evidencebased meta-analysis. Ophthalmology. 2010;117:1300-1305.

6. Oliva MS, Schottman T, Gulati M. Turning the tide of corneal blindness. Indian J Ophthalmol. 2012;60(5):423-427.

7. Pascolini D, Mariotti SP. Global estimates of visual impairment 2010. Br J Ophthalmol. 2012;96:614-618.
8. Resnikoff S, Pascolini D, Etya'ale D, et al. Global data on visual impairment in the year 2002. Bull World Health Organ. 2002;82: 844-851.

9. Williams KA, Lowe MT, Bartlett CM, Kelly L, Coster DJ, editor. The Australian corneal graft registry 2012 report. Flinders University Press. 2012:12-17. Available from: http://dspace.flinders.edu.au/xmlui/bitstream/ handle/2328/25860/Dspace\%20version\%202012\%20REPORT\%20 COMPLETE.pdf? sequence=1. Accessed, August 10, 2014

10. NHS. Survival rates following transplantation. 2014. Available at: http://www.organdonation.nhs.uk/statistics/transplant_activity_report/ current_activity_reports/ukt/activity_report_2013_14.pdf. Accessed August 10, 2014.

11. Williams KA, Lowe M, Bartlett C, Kelly TL, Coster DJ, All Contributors. Risk factors for a human corneal graft failure within the Australian corneal graft registry. Transplantation. 2008;86(12):1720-1724.

12. Ilari L, Daya SM. Long-term outcomes of keratolimbal allograft for the treatment of severe ocular surface disorders. Ophthalmology. 2002;109(7):1278-1284.

13. Solomon A, Ellies P, Anderson DF, et al. Long-term outcome of keratolimbal allograft with or without penetrating keratoplasty for total limbal stem cell deficiency. Ophthalmology. 2002;109(6): 1159-1166.

14. Barber JC. Design of a Retainable Keratoprosthesis: History, Design and Evaluation in Cats. 1st ed. Bloomington, USA: Author House; 2011.

15. Gomma A, Comyn O, Liu C. Keratoprostheses in clinical practice - a review. Clin Experiment Ophthalmol. 2010;38(2):211-214.

16. Chirila TV, Hicks CR, Dalton PD, Vijayasekaran S, Lou X, Anthony YH. Artificial cornea. Prog Polym Sci. 1998;23(3):447-473.

17. Stone W, Herbert E. Experimental study of plastic materials as replacement for the cornea. Am J Ophthalmol. 1953;36:168-173.

18. Stone W Jr. Alloplasty in surgery of the eye. N Engl J Med. 1958;258: 486-490.

19. Cardona H. Mushroom transcorneal keratoprosthesis (bolt and nut). Am J Ophthalmol. 1969;68(4):604-612.

20. Choyce DP. Evolution of Choyce 2-piece multistage perforating keratoprosthesis technique: 1967-1978. Ann Ophthalmol. 1980;12: 740-743.

21. Dohlman CH, Schneider HA, Doane MG. Prosthokeratoplasty. Am J Ophthalmol. 1974;77(5):694-700.

22. Ruedemann AD Jr. Silicone keratoprosthesis. Trans Am Ophthalmol Soc. 1974;72:329-360.

23. Cardona H. Prosthokeratoplasty. Cornea. 1983;2:179-183.

24. Girard LJ. Keratoprosthesis. Cornea. 1983;2:207-224.

25. Polack FM. Clinical results with a ceramic keratoprosthesis. Cornea. 1983;2(3):185-196.

26. Caldwell DR. The soft keratoprosthesis. Trans Am Ophthalmol Soc. 1997;95:751-802.

27. Singh D. Keratoprosthesis. Indian J Ophthalmol. 1984;32:405-407.

28. Pintucci S, Pintucci F, Cecconi M, Caiazza S. New dacron tissue colonisable keratoprosthesis: clinical experience. $\mathrm{Br} J$ Ophthalmol. 1995;79(9):825-829.

29. Pintucci S, Pintucci F, Caiazza S, Cecconi M. The dacron felt colonizable keratoprosthesis: after 15 years. Eur J Ophthalmol. 1996;6(2): 125-130.

30. Barber JC. Keratoprostheses: past present and future. Int Ophthalmol Clin. 1988;28(2):103-109.

31. Legeais J-M, Rossi C, Renard G, Salvoldelli M, D'Hermies F, Pouliquen YJ. A new flurorocarbon for keratoprosthesis. Cornea. 1992; 11(6):538-545.

32. Crawford GJ, Constable IJ, Chirila TV, Vijayasekaran S, Thompson DE. Tissue interaction with hydrogel sponges implanted in the rabbit cornea. Cornea. 1993;12(4):348-357.

33. Mester U. Experiences in animal experiments with hydrogel keratoprosthesis of different water content (abstract). Ophthalmologica. 1979; 179(1):62-69.

34. Kain HL. The development of the silicone-carbon keratoprosthesis. Refract Corneal Surg. 1993;9:209-210. 
35. Hicks CR, Filton JH, Chirila TV, Crawford GJ, Constable IJ. Keratoprostheses: advancing toward a true artificial cornea. Surv Ophthalmol. 1997;42(2):175-189.

36. Lam FC, Liu C. The future of keratoprostheses. Br J Ophthalmol. 2011; 95(3):304-305.

37. Avadhanam VS, Liu CS. Keratoprostheses: are we there yet? $\mathrm{Br} J$ Ophthalmol. 2014;98(7):849.

38. Hille K, Grabner G, Liu C, et al. Standards for modified osteo-odontokeratoprosthesis (OOKP) surgery according to Strampelli and Falcinelli: the Rome-Vienna protocol. Cornea. 2005;24:895-908.

39. de Araujo AL, Charoenrook V, de la Paz MF, Temprano J, Barraquer RI, Michael R. The role of visual evoked potential and electroretinography in the preoperative assessment of osteo-keratoprosthesis or osteo-odontokeratoprosthesis surgery. Acta Ophthalmol. 2012;90:519-525.

40. Liu C, Paul B, Tandon R, et al. The osteo-odontokeratoprosthesis (OOKP). Semin Ophthalmol. 2005;20:113-128.

41. Busuttil A, Herold J, Liu C. Psychological factors and complications in OOKP assessment and surgery. Acta Ophthalmol. 2012;90:0.

42. Busuttil A, Turton E, Herold J, Liu C. An update on patient selection for OOKP surgery; psychosocial assessment. Acta Ophthalmol. 2011; 89:0.

43. Avadhanam V, Liu C. Preventing complications of OOKP surgery. Acta Ophthalmol. 2013;91:0.

44. Garg R, Khanna P, Sinha R. Perioperative management of patients for osteo-odonto-kreatoprosthesis under general anaesthesia: a retrospective study. Indian J Anaesth. 2011;55:271-273.

45. Chirila TV. An overview of the development of artificial corneas with porous skirts and the use of pHEMA for such an application. Biomaterials. 2001;22:3311-3317.

46. Hicks CR, Crawford GJ, Dart JK, et al. AlphaCor: clinical outcomes. Cornea. 2006;25(9):1034-1042.

47. Chirila TV, Vijayasekaran S, Horne R, et al. Interpenetrating polymer network (IPN) as a permanent joint between the elements of a new type of artificial cornea. J Biomed Mater Res. 1994;28:745-753.

48. Jiraskova N, Rozsival P, Burova M, Kalfertova M. AlphaCor artificial cornea: clinical outcome. Eye. 2011;25:1138-1146.

49. Ngakeng V, Hauck MJ, Price MO, Price FW Jr. AlphaCor keratoprosthesis: a novel approach to minimize the risks of longterm postoperative complications. Cornea. 2008;27(8):905-910.

50. Hollick EJ, Watson SL, Dart JKG, Luthert PJ, Allan BDS. Legeais BioKpro III keratoprosthesis implantation: long term results in seven patients. Br J Ophthalmol. 2006;90:1146-1151.

51. Dohlman C, Harissi-Dagher M. The Boston keratoprosthesis: a new thread less design. Digital J Opthalmol. 2007;13:3.

52. Boston KPro News, Summer 2013, no 9. Available from: www.masseyeandear.org. Accessed August 21, 2014.

53. Traish AS, Chodosh J. Expanding application of the Boston type I keratoprosthesis due to advances in design and improved post-operative therapeutic strategies. Semin Ophthalmol. 2010;25(5-6):239-243.

54. Colby KA, Koo EB. Expanding indications for the Boston keratoprosthesis. Curr Opin Ophthalmol. 2011;22(4):267-273.

55. Khan BF, Harissi-Dagher M, Khan DM, Dohlman CH. Advances in Boston keratoprosthesis: enhancing retention and prevention of infection and inflammation. Int Ophthalmol Clin. 2007;47(2):61-71.

56. Zerbe BL, Belin MW, Ciolino JB; Boston Type 1 Keratoprosthesis Study Group. Results from the multicenter Boston type 1 keratoprosthesis study. Ophthalmology. 2006;113:1779-1784.

57. Greiner MA, Li JY, Mannis MJ. Longer-term vision outcomes and complications with the Boston Type 1 keratoprosthesis at the University of California, Davis experience. Ophthalmology. 2011;118(8):1543-1550.

58. Aldave AJ, Sangwan VS, Basu S, et al. International results with the Boston type I keratoprosthesis. Ophthalmology. 2012;119(8):1530-1538.

59. Chew HF, Ayres BD, Hammersmith KM, et al. Boston keratoprosthesis outcomes and complications. Cornea. 2009;28:989-996.

60. Aldave AJ, Kamal KM, Vo RC, Yu F. The Boston type I keratoprosthesis: improving outcomes and expanding indications. Ophthalmology. 2009;116(4):640-651.
61. Bradley JC, Hernandez EG, Schawb IR, Mannis MJ. Boston type I keratoprosthesis: the University of California Davis experience. Cornea. 2009;28(3):321-327.

62. Stacy RC, Jakobiec FA, Michaud NA, Dohlman CH, Colby KA. Characterization ofretrokeratoprosthetic membranes in the Boston type 1 keratoprosthesis. Arch Ophthalmol. 2011;129(3):310-316.

63. Magalhães FP, Sousa LB, Oliveira LA. Boston type I keratoprosthesis: review. Arq Bras Oftalmol. 2012;75(3):218-222.

64. Kamyar R, Weizer JS, de Paula FH, et al. Glaucoma associated with Boston type I keratoprosthesis. Cornea. 2012;31(2):134-139.

65. Banitt M. Evaluation and management of glaucoma after keratoprosthesis. Curr Opin Ophthalmol. 2011;22:133-136.

66. Li JY, Greiner MA, Brandt MC, Lim MC, Mannis MJ. Long-term complications associated with glaucoma drainage devices and Boston keratoprosthesis. Am J Ophthalmol. 2011;152(2):209-218.

67. Rivier D, Paula JS, Kim E, Dohlman CH, Grosskreutz CL. Glaucoma and keratoprosthesis surgery: role of adjunctive cyclophotocoagulation. J Glaucoma. 2009;18:321-324.

68. Robert MC, Moussally K, Harissi-Dagher M. Review of endophthalmitis following Boston keratoprosthesis type 1. Br J Ophthalmol. 2012;96(6):776-780.

69. Nouri M, Terada H, Alfonso EC, Foster CS, Durand ML, Dohlman CH. Endophthalmitis after keratoprosthesis: incidence, bacterial causes, and risk factors. Arch Ophthalmol. 2001;119(4):484-489.

70. Durand ML, Dohlman CH. Successful prevention of bacterial endophthalmitis in eyes with the Boston keratoprosthesis. Cornea. 2009;28(8):896-901.

71. Ray S, Khan BF, Dohlman CH, D'Amico DJ. Management of vitreoretinal complications in eyes with permanent keratoprosthesis. Arch Ophthalmol. 2002;120:559-566.

72. Boston KPro News, Fall 2011, no 8. Available from: www.masseyeandear.org. Accessed January 23, 2013.

73. Pujari S, Siddique SS, Dohlman CH, Chodosh J. The Boston keratoprosthesis type II: the Massachusetts eye and ear infirmary experience. Cornea. 2011;30(12):1298-1303.

74. Sivaraman KR, Hou JH, Allenmann N, de la Cruz J, Cortina MS. Retroprosthetic membrane and risk of sterile keratolysis in patients with type I Boston Keratoprosthesis. Am J Ophthalmol. 2013;155(5): 814-822.

75. Todani A, Ciolino JB, Ament JD, et al. Titanium back plate for a PMMA keratoprosthesis: clinical outcomes. Graefes Arch Clin Exp Ophthalmol. 2011;249(10):1515-1518.

76. Cruzat A, Shukla A, Dohlman CH, Colby K. Wound anatomy after type 1 Boston KPro using oversized backplates. Cornea. 2013;32(12): 1531-1536.

77. Kang JJ, Allenmann N, Cruz JD, Cortina MS. Serial Analysis of Anterior Chamber Depth and Angle Status Using Anterior Segment Optical Coherence Tomography After Boston Keratoprosthesis. Cornea. Epub 2013 Aug 22.

78. Shapiro BL, Cortés DE, Chin EK, et al. High-resolution spectral domain anterior segment optical coherence tomography in type 1 Boston keratoprosthesis. Cornea. 2013;32(7):951-955.

79. Arafat SN, Shukla AN, Dohlman CH, Chodosh J, Ciolino JB, editors. Cross-linking donor corneas for the Boston Keratoprosthesis: a method of increasing resistance to collagenolytic degradation. Poster session presented at: ARVO 2012; May 10; 2012; Florida, USA.

80. Cruzat A, Tauber A, Shukla A, Paschalis EL, Pineda R, Dohlman CH. Low-cost and readily available tissue carriers for the Boston keratoprosthesis: a review of possibilities. J Ophthalmol. 2013;2013:5.

81. Kim MJ, Yu F, Aldave AJ. Microbial keratitis after Boston type 1 keratoprosthesis implantation: incidence, organisms, risk factors, and out comes. Ophthalmology. 2013;120(11):2209-2216.

82. Todani A, Behlau I, Fava MA, et al. Intraocular pressure measurement by radio wave telemetry. Invest Ophthalmol Vis Sci. 2011;52(13): 9573-9580.

83. Melki S, Todani A, Cherfan G. An implantable intraocular pressure transducer: initial safety outcomes. JAMA Ophthalmol. 2014;132:1221-1225. 
84. León CR, Barraquer JI Jr, Barraquer JI Sr. Coralline hydroxyapatite keratoprosthesis in rabbits. J Refract Surg. 1997;13(1):74-78.

85. Strampelli B. Keratoprosthesis with osteodontal tissue. Am J Ophthalmol. 1963;89:1029-1039.

86. Casey TA. Osteo-odonto-keratoprosthesis. Proc R Soc Med. 1966;59: 530-531.

87. Temprano J. Keratoprosthesis with tibial autograft. Refract Corneal Surg. 1993;9:192-193.

88. Falcinelli GC, Barogi G, Caselli M, Colliardo P, Taloni M. Personal changes and innovations in Strampelli's osteo-odontokeratoprosthesis. Anales Del Instituto Barraquer (Barc). 1999;28(S):47-48.

89. Tan A, Tan DT, Tan XW, Mehta JS. Osteo-odonto keratoprosthesis: systematic review of surgical outcomes and complication rates. Ocul Surf. 2012;10(1):5-25.

90. Liu C, Okera S, Tandon R, Herold J, Hull C, Thorp S. Visual rehabilitation in end-stage inflammatory ocular surface disease with the osteoodontokeratoprosthesis: results from the UK. Br J Ophthalmol. 2008; 92:1211-1217.

91. Marchi V, Ricci R, Pecorella I, Ciardi A, Di Tondo U. Osteo-odontokeratoprosthesis. Description of surgical technique with results in 85 patients. Cornea. 1994;13:125-130.

92. Falcinelli G. Modified osteo-odonto-keratoprosthesis for treatment of corneal blindness: long-term anatomical and functional outcomes in 181 cases. Arch Ophthalmol. 2005;123:1319-1329.

93. Michael R, Charoenrook V, de la Paz MF, Hitzl W, Temprano J, Barraquer RI. Long-term functional and anatomical results of osteoand osteoodonto-keratoprosthesis. Graefes Arch Clin Exp Ophthalmol. 2008;246:1133-1137.

94. Hille K, Hille A, Ruprecht KW. Medium term results in keratoprostheses with biocompatible and biological haptic. Graefes Arch Clin Exp Ophthalmol. 2006;244:696-704.

95. Iyer G, Pillai VS, Srinivasan B, et al. Modified osteo-odonto keratoprosthesis-the Indian experience-results of the first 50 cases. Cornea. 2010;29:771-776.

96. De La Paz MF, De Toledo JÁ, Charoenrook V, et al. Impact of clinical factors on the long-term functional and anatomic outcomes of osteoodonto-keratoprosthesis and tibial bone keratoprosthesis. Am J Ophthalmol. 2011;151(5):829-839.
97. Avadhanam V, Liu C. Managing laminar resorption in Osteo-odontokeratoprosthesis. Am J Ophthalmol. 2014;158(2):213-214.

98. Stoiber J, Forstner R, Csáky D, Ruckhofer J, Grabner G. Evaluation of bone reduction in osteoodontokeratoprosthesis (OOKP) by threedimensional computed tomography. Cornea. 2003;22(2):126-130.

99. Sipkova Z, Lam FC, Francis I, Herold J, Liu C. Serial 3-dimensional computed tomography and a novel method of volumetric analysis for the evaluation of the osteo-odonto-keratoprosthesis. Cornea. 2013; 32(4):401-406.

100. Kumar RS, Tan DT, Por YM, et al. Glaucoma management in patients with osteo-odonto-keratoprosthesis (OOKP). The Singapore OOKP study. J Glaucoma. 2009;18(5):354-360.

101. Falcinelli GC, Falsini B, Taloni M, Piccardi M, Falcinelli G. Detection of glaucomatous damage in patients with osteo-odontokeratoprosthesis. Br J Ophthalmol. 1995;79(2):129-134.

102. Hughes EH, Mokete B, Ainsworth G, et al. Vitreoretinal complications of osteo-odonto keratoprosthesis surgery. Retina. 2008;28:1138-1145.

103. Lim LS, Ang CL, Wong E, Wong DW, Tan DT. Vitreoretinal complications and vitreoretinal surgery in osteo-odonto-keratoprosthesis surgery. Am J Ophthalmol. 2014;157(2):349-354.

104. Avadhanam VS, Herold J, Thorp S, Liu CS. Mitomycin-C for mucous membrane overgrowth in OOKP eyes. Cornea. 2014;33:981-984.

105. Kim MK, Lee SM, Lee JL, et al. Long-term outcome in ocular intractable surface disease with Seoul-type keratoprosthesis. Cornea. 2007; 26(5):546-551.

106. Iakymenko S. Forty-five years of keratoprosthesis study and application at the Filatov Institute: a retrospective analysis of 1060 cases. Int J Ophthalmol. 2013;6(3):375-380.

107. Huang Y, Yu J, Liu L, Du G, Song J, Guo H. Moscow eye microsurgery complex in Russia keratoprosthesis in Beijing. Ophthalmology. 2011;118(1):41-46.

108. Ghaffariyeh A, Honarpisheh N, Karkhaneh A, et al. Fyodorov-Zuev keratoprosthesis implantation: long-term results in patients with multiple failed corneal grafts. Grafes Arch Clin Exp Ophthalmol. 2011; 249(1):93-101.

109. Hicks CR, Crawford GJ, Lou X, et al. Corneal replacement using a synthetic hydrogel cornea, AlphaCor[trade]: device, preliminary outcomes and complications. Eye. 2003;17(3):385-392.
Clinical Ophthalmology

\section{Publish your work in this journal}

Clinical Ophthalmology is an international, peer-reviewed journal covering all subspecialties within ophthalmology. Key topics include: Optometry; Visual science; Pharmacology and drug therapy in eye diseases; Basic Sciences; Primary and Secondary eye care; Patient Safety and Quality of Care Improvements. This journal is indexed on Submit your manuscript here: http://www.dovepress.com/clinical-ophthalmology-journal

\section{Dovepress}

PubMed Central and CAS, and is the official journal of The Society of Clinical Ophthalmology (SCO). The manuscript management system is completely online and includes a very quick and fair peer-review system, which is all easy to use. Visit http://www.dovepress.com/ testimonials.php to read real quotes from published authors. 